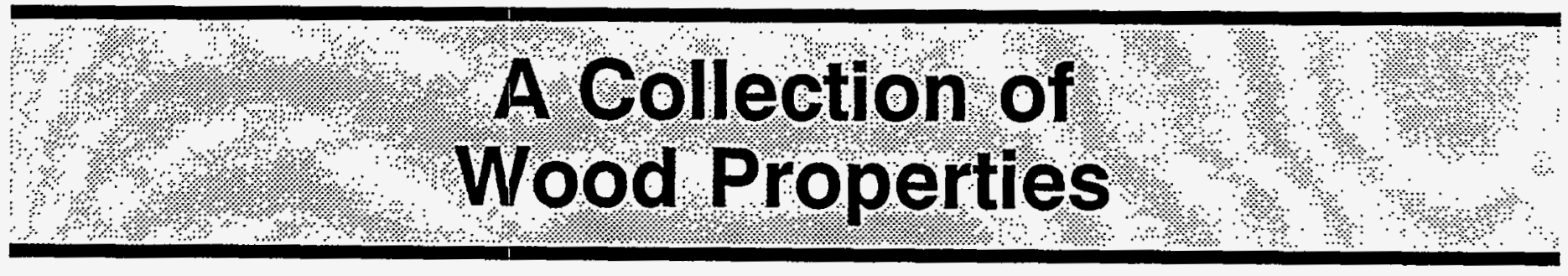

\title{
Version 1.1
}

September 1992

by

Richard L. Toennisson

Wood Products Engineer

Forest Resources Development Program

Tennessee Valley Authority

Norris, Tennessee 37828-2001

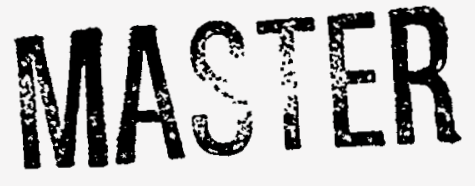

DSSTRIBUTION OE IHIS DERUMENI IS UIVLIMITED 
TVA is an equal opportunity employer, and is committed to ensuring that the benefits of programs receiving TVA financial assistance are available to all eligible persons regardless of race, color, national origin, handicap, or age. 


\section{DISCLAIMER}

This report was prepared as an account of work sponsored by an agency of the United States Government. Neither the United States Government nor any agency thereof, nor any of their employees, make any warranty, express or implied, or assumes any legal liability or responsibility for the accuracy, completeness, or usefulness of any information, apparatus, product, or process disclosed, or represents that its use would not infringe privately owned rights. Reference herein to any specific commercial product, process, or service by trade name, trademark, manufacturer, or otherwise does not necessarily constitute or imply its endorsement, recommendation, or favoring by the United States Government or any agency thereof. The views and opinions of authors expressed herein do not necessarily state or reflect those of the United States Government or any agency thereof. 


\section{DISCLAIMER}

Portions of this document may be illegible in electronic image products. Images are produced from the best available original document. 


\section{CONTENTS}

Page

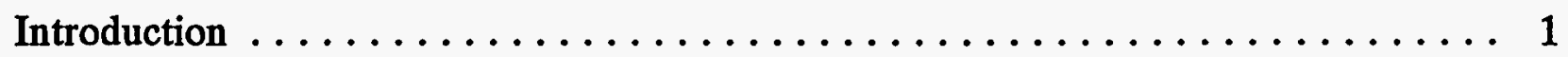

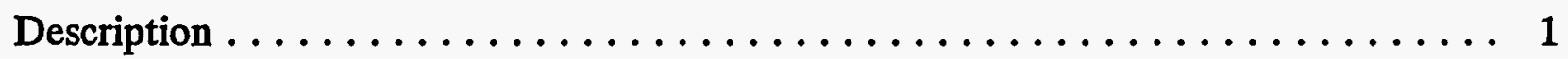

Table 1. Wood Property Index Classes $\ldots \ldots \ldots \ldots \ldots \ldots \ldots \ldots \ldots$

Recommended Use $\ldots \ldots \ldots \ldots \ldots \ldots \ldots \ldots \ldots \ldots \ldots \ldots \ldots \ldots$

Additions or Corrections $\ldots \ldots \ldots \ldots \ldots \ldots \ldots \ldots \ldots \ldots \ldots \ldots \ldots \ldots \ldots \ldots$

Table 2. File WOODF: Properties of the World's Woods $\ldots \ldots \ldots \ldots \ldots \ldots \ldots$

Table 3. File WOODP: Properties of Commonly Used Woods . . . . . . . . . 12

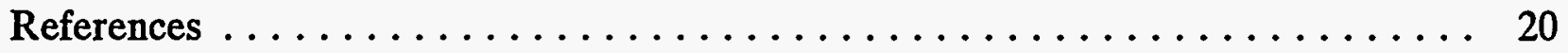

Glossary .................................. 21 


\section{INTRODUCTION}

Reference books are the primary source of wood property data for use in research, school, or business. It has been our experience that obtaining the needed data from several references can be a cumbersome and time-consuming ordeal. These spreadsheets are a compilation of data from several well-known references. Version 1.1 includes the most recent update of commercial woods of the United States and the world. It is intended that they be used for quick summaries, comparisons, and calculations to increase the efficiency of the professional user in report preparation.

\section{DESCRIPTION}

The information is divided in two parts which are stored on separate Lotus 1-2-3 spreadsheet files (diskette is enclosed). The first file is a summary of the basic properties of 279 commercial lumber species from around the world. The data appearing in this file, named WOODF (See Table 2, page 4), are: common names, specific gravities, volumetric shrinkage, calculated weights, bending strength, compression, hardness, stiffness, shock resistance, drying ease, machinability, durability, color, scientific name, and world origin. The second file, named WOODP (See Table 3, page 12), contains more detailed information for 149 domestic species and selected imports. It contains all of the data appearing in the world species file, except for color, plus the following: green moisture contents, Btu values, shrinkage values and calculations, heat values and calculations, weight and dimension estimates, and a more detailed summary of relative wood strength and manufacturing characteristics. 
The wood properties appear as values rated from 1 to 5 based on mechanical property index classes published by Panshin and de Zeeuw, 1970. In general, these values may be considered a rating from good $=1$, to poor $=5$; or hard $=1$, to soft $=5$. Other important properties have been given similar numeric ratings. Therefore, drying ease has only 3 ratings based on the complexity of drying; so a rating of 1 may warp, a 2 may warp and dry slow, and a 3 may check, warp, and dry slow. Machinability has ratings from $1=$ good, to 3 = poor, and wood with rating 4 contains silicon. Durability has 6 ratings from $1=$ very durable to $6=$ not durable. Gluing ease has 4 ratings from $1=$ good, to $4=$ poor. $\mathrm{A}+$ preceding a rating indicates a high value within that rating group. The following table (adapted from Panshin and de Zeeuw, 1970) further describes the value ratings.

Table 1. Wood Property Index Classes

\begin{tabular}{|c|c|c|c|c|c|c|c|}
\hline \multirow{2}{*}{$\begin{array}{c}\text { WOOD } \\
\text { PROPERTY }\end{array}$} & \multirow{2}{*}{ W } & \multicolumn{6}{|c|}{ \% \% $\%$ IINDEX GISASES $\% \%$} \\
\hline & & 60 & $\%$ & +4: & 3 & 22 & 1\%: \\
\hline Bending Strength & Rupture modulus in bending, green wood, psi & & $<6000$ & 6000 to & 7000 to & 8000 to & $11,000+$ \\
\hline Compression & Max. crushing strength parallel, green wood, psi & & $<2200$ & 2200 to & 3000 to & 3600 to & $4500+$ \\
\hline Hardness & Side Hardness, green wood, lb. & & $<400$ & 400 to & 600 to & 900 to & $1200+$ \\
\hline Stiffness & Elasticity modulus in bending, psi x 1000 & & $<800$ & 800 to & 1000 to & 1300 to & $1500+$ \\
\hline Shock Resistance & Height of drop with $50 \mathrm{lb}$. hammer, inches & & 15 to & 25 to & 35 to & 50 to & $70+$ \\
\hline Splitting & Load, lb/in. of width to split green wood & & $<150$ & 150 to & 250 to & 350 to & $450+$ \\
\hline Screw Holding & Screw withdrawal as function of sp. gr. OD & & $<0.40$ & 0.40 to & 0.45 to & 0.55 to & $0.70+$ \\
\hline Drying Ease & Number of drying problems & & & & 3 & 2 & 1 \\
\hline Machinability & Quality & & & Silicon & Poor & Medium & Good \\
\hline Durability & Degree of resistance to decay & None & Very Low & Low & Moderate & Mod. Res. & Resistant \\
\hline Gluing Ease & Quality & & & Difficult & Not Well & Well & Easily \\
\hline
\end{tabular}

\section{RECOMMENDED USE}

It is not intended that all of the material in the spreadsheet tables be used in a single job. The user should delete the material in those columns or rows which are not wanted 
or distract from the final form of the report. The user should take care not to save the edits to the original computer file. It is recommended that nothing ever be saved unless it is to another file.

The files are write protected, except for the highlighted, calculable values. It is possible to change these highlighted numbers to any desired value, therefore tailoring the table to specific needs. The highlighted, changeable values are: moisture content, board dimensions, volume of lumber, and wood and bark percentages. When a highlighted value is changed all of the values in the table which use it are recalculated. An example of each file follows with some commonly used values inserted in the highlighted fields.

Note: In order to change or edit any copy of Wood Properties the write protect must be removed. Selection of 'disengage' in 'Global' under 'Worksheet' will remove the protection. Selection of 'engage' will return the protection. It is recommended that write protection always be in place except while editing.

\section{ADDITIONS or CORRECTIONS}

We request that users contact the author to complete missing information, add new species, or make suggestions.

Rick Toennisson

TVA Forestry Building

17 Ridgeway Road

Norris, TN 37828-2001 


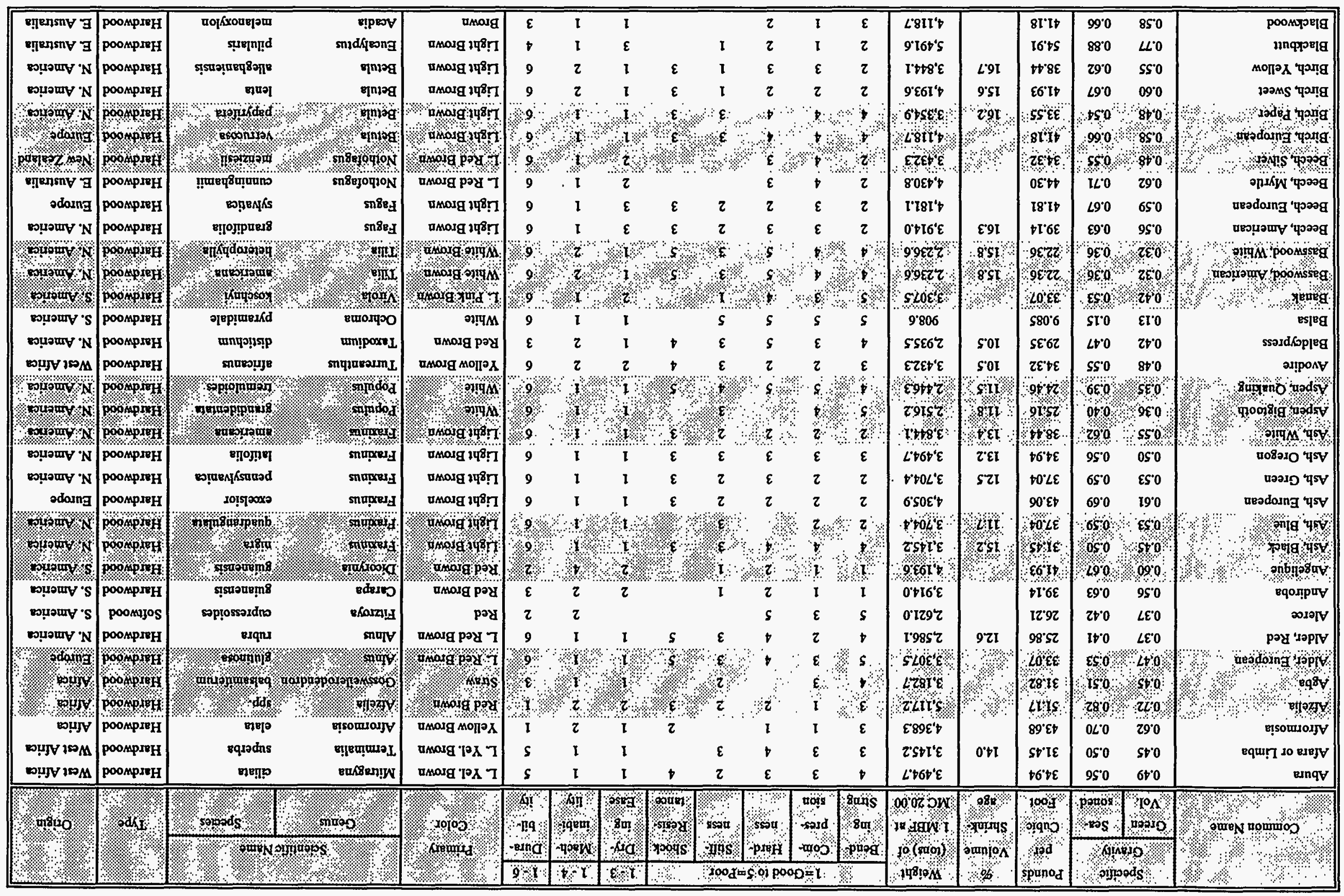




\begin{tabular}{|c|c|c|c|c|c|c|c|c|c|c|c|c|c|c|c|c|c|c|}
\hline \multirow{3}{*}{ कैo } & \multirow{2}{*}{\multicolumn{2}{|c|}{$\begin{array}{l}\text { Spectic } \\
\text { oravity : }\end{array}$}} & \multirow{3}{*}{$\begin{array}{l}\text { Pounds } \\
\text { per } \\
\text { Ciutic } \\
\text { Foot }\end{array}$} & \multirow{3}{*}{$\begin{array}{l}\text { of } \\
\text { Volutre } \\
\text { Shrink } \\
\text { onge }\end{array}$} & \multirow{3}{*}{$\begin{array}{l}\text { Weight } \\
\text { (tons) of } \\
\text { MBF at } \\
\text { MQ } 2000\end{array}$} & \multicolumn{5}{|c|}{ १४ $1=$ Good to $5=$ Poor } & \multirow{3}{*}{$\begin{array}{l}1 \% 3 \\
\text { Diy } \\
\text { ing } \\
\text { Eise. }\end{array}$} & \multirow{3}{*}{ 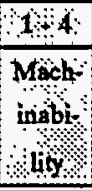 } & \multirow{3}{*}{ 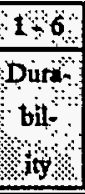 } & \multirow{3}{*}{ Pringl } & \multirow{2}{*}{\multicolumn{2}{|c|}{ 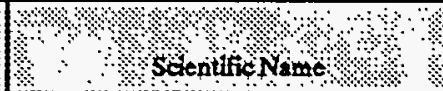 }} & \multirow{3}{*}{ (1) } & \multirow{3}{*}{ orsin } \\
\hline & & & & & & \multirow{2}{*}{$\begin{array}{l}\text { Bends } \\
\text { tins } \\
\text { Strno }\end{array}$} & \multirow{2}{*}{ cotrs } & \multirow{2}{*}{$\begin{array}{r}\text { Had: } \\
\text { nes: } \\
\end{array}$} & \multirow{2}{*}{$\begin{array}{l}\text { Stity } \\
\text { ness } \\
\end{array}$} & \multirow{2}{*}{$\begin{array}{l}\text { Shock } \\
\text { Rests: } \\
\text { topece }\end{array}$} & & & & & & & & \\
\hline & Golect & soned & & & & & & & & & & & & & istas & $\$$ & & \\
\hline Blackwood, African & 1.06 & 1.20 & 74.88 & & $7,488.6$ & & & 1 & & & 2 & 1 & 2 & Diach & Daldet & & od & East Africa \\
\hline Boxwood & 0.80 & 0.91 & 56.78 & & $5,678.9$ & & & & & & 3 & 3 & 3 & L. Yellow & 3uxus & rens & ardwood & Europe/Asia \\
\hline West Indian & 0.75 & 0.85 & 53.04 & & $5,304,4$ & & & & & & 3 & 3 & 3 & L. Yellow & Gossypiosperm & pracos & lardwood & S. America \\
\hline Brush Box & 0,79 & 0.90 & $56: 16$ & $\$$ & $\$, 616,4$ & 2 & 1 & 1 & 1 & & 3 & 3 & 4 & Brown & Tristania & oonerts & Hardwood & EAustrifin \\
\hline ibongn & 079 & 088 & $\$ 4.91$ & 144 & 54916 & 3 & & $i$ & $\therefore$ & & 2 & 1 & 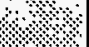 & Brown & \% & $s p p$ & Hardwood & West Afros \\
\hline Biickeye, Yelow & 0.33 & 0.37 & 23.05 & 12,5 & 2,3005 & 3 & 5 & 5 & 4 & 5 & 1 & 2. & 5 & Yelow White & Aescultus & octadra & Harowod & $\mathrm{NAn+10a}$ \\
\hline Butternut & 0.36 & 0.40 & 25.16 & 10.6 & $2,516.2$ & 4 & 4 & 5 & 4 & 5 & 1 & 2 & 6 & Brown & Juglans & cinerea & Hardwood & N. America \\
\hline talpa, & 0.38 & 0.43 & 26.56 & 7.3 & $2,656.0$ & 4 & 4 & 4 & 4 & 3 & 1 & 2 & 2 & White & pa & spec & $\infty$ & N. As \\
\hline icil & 0.51 & 0.58 & 36.19 & & $3,619.5$ & 4 & 3 & 2 & 5 & & 2 & 1 & 2 & Red Brown & uniperus & procera & & East \\
\hline Cedar Llas L & 042 & 0.47 & 29,35 & 92 & 2,935 & $4:$ & 3 & 4 & 3 & 4 & 1 & 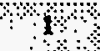 & 2 & Yoxollow & Champospanis & 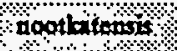 & fwood & NAnerca \\
\hline Cedar, Allantio White & 0,31 & 0,35 & 2167 & 8,8 & 21667 & 8 & 4 & 5 & 5 & 5 & 1 & 2 & 2 & White & Chamaerypant & $\%$ & soltood & $\mathrm{N}$ homnch \\
\hline Codar Inouse $\$$ & 0.35 & 0.39 & 24,46 & 7.6 & 2,4463 & 4 & 3 & 3 & 4 & s. & 1 & 2 & 2 & $1 /$ Red Brown & $1: 16=0$ dro & 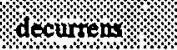 & 01000 a & Nonneric \\
\hline ebanon & 0.49 & 0.56 & 34.94 & & $3,494.7$ & & & 5 & 5 & & & 1 & 1 & Light Brown & Cedrus & & ood & inor \\
\hline 年 & 0.29 & 0.32 & 20.27 & 7.2 & 5.9 & 5 & 5 & 5 & 5 & 5 & 1 & 2 & 2 & & & & & \\
\hline rford & 0.40 & 0.45 & 27.96 & 10.1 & 7 & 4 & 3 & 4 & 2 & 5 & 1 & 1 & 2 & L. Yel. Brown & Chamaecyparis & & ftwood & N. America \\
\hline Ceiba & 0,28 & 0.32 & 19.97 & & $1,997,0^{\circ}$ & $\$$ & $\$$ & & s. & & 3 & 2 & 6 & strat & Cobs & peptanda & Hard $06 \mathrm{~d}$ & Wert Africh \\
\hline Chonchen & 036 & 0.41 & 25,58 & & $2 \$ 58.6$ & 5 & 4 & & 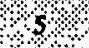 & & 2 & 2 & 6 & Sirat & Antury & frichin & Hordwod & Wat Anich \\
\hline $\mathrm{y}, \mathrm{Black}$ & 0.47 & 0,53 & 3285 & 115 & 3285,0 & 3 & 3 & 3 & 2 & 4 & 1 & 2 & 2 & Red Brow/ & Prinus & 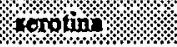 & Hardood & $N /$ nenci \\
\hline Cherry, Eur & 0.54 & 0.61 & 38.06 & & $3,806.7$ & 2 & 3 & 3 & 2 & 4 & 1 & 2 & 5 & Brown & 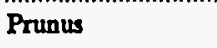 & & & \\
\hline & 0.40 & 0.45 & 27.96 & 11.6 & $2,795.7$ & 4 & 4 & 4 & 4 & 4 & 1 & 1 & 2 & rown & & & & N. \\
\hline & 0.48 & 0.55 & 34.32 & & $3,432.3$ & 3 & 4 & 4 & 4 & 4. & 2 & & 3 & Light Brown & Castanea & sativa & yood & Europe \\
\hline quito Aln & 0.74 & & 46,00 & & १ু। & \% & & & & & & 2 & & Hoht Btowf & Diplotropts & martwo & 1rowod & SHantra \\
\hline Gintanot & 0,49 & 0.56 & 34.94 & & $3,494,7$ & & & & & & & 1 & & Brown & Conisunonium & $p_{p}$ & Hardwood & $5 . \mathrm{EA}$ \\
\hline Gordio Wost Árica & 038 & 0.43 & 26,83 & & $2,683,4$ & 4 & 18 & & $\therefore$ & & 1 & 2 & 4 & Brotr: & Cordin & $a_{\mathrm{ph}} \ldots$ & Harood & $w+4$ nin \\
\hline$\dddot{c}$ & 0.31 & 0.35 & 21.67 & 10.5 & $2,166.7$ & 5 & 5 & 5 & 3 & 5 & 2 & 3 & 6 & White & & balsam & Hardwood & N. America \\
\hline & 0.31 & 0.35 & 21.67 & 12.4 & $2,166.7$ & 5 & 4 & 5 & 2 & 5 & 2 & 3 & 6 & hite & & Irpa & ood & rica \\
\hline Cotto & 0.37 & 0.41 & 25.86 & 14.1 & $2,586,1$ & 5 & 4 & 5 & 3 & 5 & 2 & 3 & 6 & White & Populus & aertoraes & ardwood & N. America \\
\hline peodar & 0.49 & 0.56 & 34.94 & ? & 3,4947 & 4 & 4 & 3 & 3 & 4 & 1 & 2 & 3 & Lght Brown & $c \alpha$ & doodar & Softwoo & Contrifsis \\
\hline Dogyood Flowering & 0.64 & 0.72 & 44.73: & 20.8 & 473.2 & 2 & 2 & $\%$ & 3 & 2 & 1 & 2 & 6 & Light Btown & Connus & & Hardwood & N America \\
\hline Douglas fin & 0,45 & 0,50 & 3145 & 107 & $3,145,2$ & 3 & $3:$ & 4 & 2 & 3 & 11 & $2 \%$ & 4 & Rked Buwn & Psetdoftuga & neppestil & $61+0 \%$ ot & N. Anerich \\
\hline Ebony, African & 0.89 & 1.01 & 63.02 & & $6,302.9$ & 1 & 1 & +1 & +1 & +1 & 1 & 4 & & Black & Diospyros & crassifora & Hardwood & West Africa \\
\hline Ebony, Borneo & 0.77 & 0.87 & 54.29 & & $5,429.2$ & 1 & 1 & +1 & +1 & & 2 & 4 & 2 & $\mathrm{rn} / \mathrm{BlkStp}$ & Diospyros & spp. & rood & S. E. Asia \\
\hline Ebony, East Indian & 1.03 & 1.17 & 73.01 & & $7,301.4$ & & 1 & +1 & +1 & & 2 & 4 & 2 & Black & Diospyros & ebenum & lardwood & India \\
\hline Ekko or Azobe " & 092 & 1.04 & 64.9 & & 6,4001 & $1 \%$ & 1 & 1 & 1 & & 3 & 3 & 1 & D. Red Brown & Tophir: & nluth & rardwood & West Arnch \\
\hline Elm; Americai & 0.46 & 0.52 & 3215 & 11,6 & $3,215,1$ & 3 & 4 & 4 & 3 & $\therefore$ & & 3 & & Light Brown. & Ulinus & americanas & Hardwood & NAmerice \\
\hline Elm English & 0.48 & 0.55 & 34.32 & $\therefore$ & 3,4323 & 3. & 4 & 3 & 3 & 2 & 2 & 3 & 6. & Light Browa: & Ulinus & proceras & Harcwood & Europe \\
\hline
\end{tabular}




\section{Table 2. (continued) Properties of the World's Woods}

\begin{tabular}{|c|c|c|c|c|c|c|c|c|c|c|c|c|c|c|c|c|c|c|}
\hline \multirow{3}{*}{ 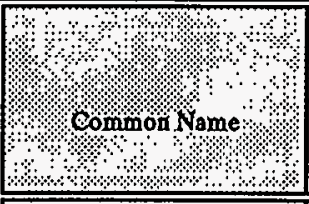 } & \multirow{2}{*}{\multicolumn{2}{|c|}{$\begin{array}{l}\text { spocifio } \\
\text { oranity }\end{array}$}} & \multirow{3}{*}{$\begin{array}{l}\text { Pounds } \\
\text { ror: } \\
\text { robis } \\
\text { root }\end{array}$} & \multirow{3}{*}{ 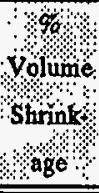 } & \multirow{3}{*}{$\begin{array}{l}\text { Woight } \\
\text { (rons) or } \\
\text { MBBF } \\
\text { MC 20.00 }\end{array}$} & \multirow{3}{*}{ Bond } & \multirow{3}{*}{$\begin{array}{l}1=0 \\
\text { Com. } \\
\text { prest: } \\
\text { sion }\end{array}$} & \multirow{3}{*}{ tird: } & \multirow{3}{*}{ soor } & \multirow{3}{*}{ Shock } & \multirow{3}{*}{$\frac{13}{\text { Diys }}$} & \multirow{3}{*}{ 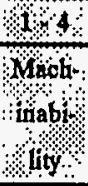 } & \multirow{3}{*}{ Dorro } & \multirow{3}{*}{ \% } & \multirow{2}{*}{\multicolumn{2}{|c|}{28 scionifific Namo }} & \multirow{3}{*}{ r., } & \multirow{3}{*}{ 3. } \\
\hline & & & & & & & & & & & & & & & & & & \\
\hline & roen: & soat & & & & & & & & & & & & & \%ै. & \% speres & & \\
\hline Elm, Japanese & 0.51 & 0.58 & 36.19 & & $3,619.5$ & 3 & 4 & 3 & 3 & 2 & 2 & 3 & 6 & Light Brown & Ulmus & laciniata & Iardwood & N.E. Asia \\
\hline Rock & 0.57 & 0.64 & 39.84 & 14.1 & $3,983.9$ & 2 & 2 & 2 & 3 & 2 & 1 & 3 & 6 & Light Brown & Ulmus & thomasii & rood & N. America \\
\hline n, Slippery & 0.48 & 0.54 & 33.55 & 13.8 & $3,354.9$ & 3 & 3 & 3 & 3 & 2 & 1 & 3 & 6 & Light Brown & Ulmus & rubra & Hardwood & N. America \\
\hline flm. Wy & $039 \%$ & 0.67 & 41,81 & ই।. & \%181 & 3 & 3. & 2 & 2 & $2 \%$ & 2 & 3. & 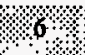 & Ligh Browh & Ulinis: & 81967 & Harowod & Europ \\
\hline Fr, Balsam & 103. & 0,38 & 23,7 & $11.2 \%$ & $23764 \%$ & 5 & & 5 & 4 & 5. & 1 & $1 \%$ & 6 & White & Sibits: & brilsanen & & Americi: \\
\hline $\mathrm{rircai}$ & $0.36 \%$ & $0 \%$ & $25.16 \%$ & 1114 & 25162 & \$ & 4 & 3 & 3 & 5 & 19 & $3 \%$ & $\% \%$ & Whitio. & Abies & maguificic & oftrood. & Hornorica. \\
\hline Fir, Grand & 0.35 & 0.39 & 24.46 & 11.0 & $2,446.3$ & 5 & 4 & 5 & 3 & 5 & 1 & 3 & 6 & White & Abies & grandis & Softwood & N. America \\
\hline Noble & 0.37 & 0.41 & 25.86 & 12.4 & $2,586.1$ & 4 & 3 & 5 & 2 & 5 & 1 & 3 & 6 & White & Abies & procera & Softwood & N. America \\
\hline , Pacific Siver & 0.40 & 0.45 & 27.96 & 13.0 & 2795.7. & 4 & 3 & 5 & 2 & 5 & 1 & 3 & 6 & White & Abies & amabilis & Softwood & N. America \\
\hline Gits subwingits & 1031. & $0.35 \%$ & $21.67 \%$ & $\%$ & $2,186.7$ & 3 & 4 & $\because$ & 3 & 3 & 19 & 3 & 6 & White & \%bies & & $8+1800 d$ & Normench \\
\hline Frow wile & 037 & $10 \% 1$ & 25.86 & 9.8 & 25861. & 5 & 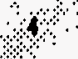 & 5 & 3 & 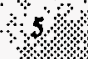 & 1 & 3 & 6 & White. & Abile: & & Sorgyood. & N A Ambrich \\
\hline Fropos. & 10.5\% & 0,01 & 3806 & ।. & $8.8067 ?$ & 2 & 2 & 2 & I. & & 18 & 1 & 3 & 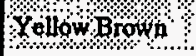 & corita & godidian: & Hard, & S.Amercr \\
\hline Gaboon & 0.38 & 0.43 & 26.83 & & $2,683.4$ & 5 & 4 & 5 & 3 & 5 & 1 & 3 & 6 & L Pink Brown & Aucoumea & klaineana & Hardwood & West Africa \\
\hline $\operatorname{lan}$ & 0.95 & & 65.00 & & & & & & & & 1 & 4 & 2 & Brown & Licania & & Hardwood & S. America \\
\hline hor & 0.48 & 0.55 & 34.32 & & 3,4323 & 4 & 3 & 3 & 2 & & 2 & 1 & 4 & Red Brown & Entandro & angolense & Hardwood & West Africa \\
\hline enheat & 4.83 & 0.93 & 58.01 & & 98012 & 1 & 1 & $\%$ & 1 & & 3 & 3. & 3 & Green & Oooter. & rodiaer & Hordwood & $S \%$ mencs \\
\hline th & $8,51$. & 0,58 & $36: 19$ & & .6 .695 & 3 & 2 & 2. & & & 2 & 18 & 4 & Ruk Brow & quarer & sedrate & $14+4,04$ & Westicos \\
\hline Sinit. $s$ & 079 & $0.0 \%$ & $56 ; 6$ & 226 & 8,6644 & 3 & 1 & 8. & १: & 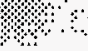 & 1 & 3 & 8 & Hel B Bown & furctsping & gotoutus & Hordwood & EAAustriti \\
\hline $\mathrm{m}_{\mathrm{m}} \mathrm{S}_{\mathrm{I}}$ & 0.89 & 1.01 & 63.02 & & $6,302.9$ & 3 & $i$ & 1 & 1 & & 3 & 2 & 4 & Light Brown & Eucalyptus & maculata & rood & E. Australia \\
\hline ney Blue & 0.72 & 0.82 & 51.17 & & $5,117.2$ & 2 & 1 & 2 & 1 & & 2 & 2 & 4 & & Eucalyptus & saligna & ood & E. Australia \\
\hline therry & 0.49 & 0.55 & 34.25 & 16.9 & 3,4248 & 4 & 4 & 3 & 4 & 3 & 1 & 1 & 6 & Light Brown & $\mathrm{Ce}$ & occidentalis & Hardwood & N. America \\
\hline Hald & 0.38 . & 0.66 & 41.18 & $1 \%$ & 41187 & 2 & 2. & 4 & a & & 1 & 1 & 6 & H Yel. Bnowa & Adno: & oonofifolit & Hardwood & India \\
\hline 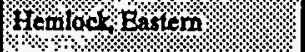 & 0,038 & 8.43: & 26.56 & \%9.7. & 2,6560 & $\%$ & 3 & & 3. & & $1 \%$ & 8 & 8 & White. & Tsuga: & caradensis & oftrood & N:Amerion \\
\hline untan & 042. & 0.4\%. & 29,35 & $111 \%$ & 2,9353 & 4. & 4 & 4 & 3 & 4 & $1 \%$ & 2 & $\%$ & Whitc. & Tsuge & mertentiano & fitwoon & NAtrenca \\
\hline $\mathrm{m}$ & 0.42 & 0.47 & 29.35 & 12.4 & $2,935.5$ & 4 & 3 & 4 & 2 & 5 & 1 & 2 & 6 & White & Tsuga & heterophylla & oftwood & N. America \\
\hline & 0.60 & 0.67 & 41.93 & 13.6 & $4,193.6$ & 2 & 1 & & 2 & 2 & 1 & 1 & 6 & th. or $\mathrm{R} . \mathrm{Bm}$ & Carya & cordiformis & rood & N. America \\
\hline Hickory, Mockernut & 0.64 & 0.72 & 44.73 & 17.8 & $4,473.2$ & 1 & 1 & & 1 & 1 & 1 & 1 & 6 & Whe or R. Brn & Carya & tomentosa & $\operatorname{cod}$ & N. America \\
\hline Hickory Wutros & 0,56 & 0.63 & 3914 & 13.6 & $\$, 014,0:$ & 2 & 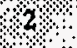 & & $\%$ & 2. & 1 & $1 \%$ & 6. & Whit or $R$ B & Cary & myt3ificion & ardwood & America \\
\hline Hicrons, Yecan & 0.60 & 0.67 & 4.93 & 13.6. & $4,193,6$ & 2 & 2 & & & 7 & 11 & & 6. & Whtor P Bm & Cayyi & illing & o.d & $\mathrm{ASmnne}$ \\
\hline 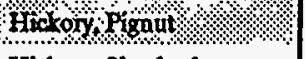 & 0.66 & $0.7 \%$ & 46.13 & 17.9 & $\% 613.0$ & 1 & $\%$ & $\therefore \cdots$ & ?. & 19 & 19 & 18 & 6. & Who orR Brm & caryo & Babra & Hardood & Nonerics. \\
\hline cory, Shagbark & 0.64 & 0.72 & 44.73 & 16.7 & $4,473.2$ & 1 & 1 & 1 & 1 & 1 & 1 & 1 & 6 & Wht or R. Bm & Carya & ova & ood & N. America \\
\hline ory, Shellbar & 0.62 & 0.69 & 43.33 & 19.2 & $4,333.4$ & 2 & 2 & & 2 & 1 & 1 & 1 & 6 & Brn & $\mathrm{Ca}$ & lacinio & ood & erica \\
\hline Eory, Water & 0.61 & 0.68 & 42.63 & 13.6 & $4,263.5$ & 1 & 1 & & 1 & 2 & 1 & 1 & 6 & Wht or R. Brm & 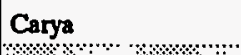 & aquatica & ardwood & N. America \\
\hline Vitinertion & 050 & 0.56 & $8,9,9$ & 16,9 & 34947 & 4 & 4 & & 4 & 2 & 2 & 1 & 8. & White & 1 & & Hordwood & H/rneoci. \\
\hline pers & 0,0 & 0.80 & 49,2 & 3 & 4,9924 & & $: \because$ & & & & 8 & 2 & 8. & White & & & & Euri \\
\hline Honeylo & 0.60 & 0,67 & 41,93 & 10.8 & $4,193,6$ & 2 & 2 & 1 . & 3 & 3 & 2 & 2 & 3. & $Y$ Bnowa & 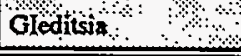 & & ood & rica \\
\hline
\end{tabular}




\begin{tabular}{|c|c|c|c|c|c|c|c|c|c|c|c|c|c|c|c|c|c|c|}
\hline \multirow{3}{*}{ + } & \multirow{2}{*}{\multicolumn{2}{|c|}{$\begin{array}{r}\text { Specific } \\
\text { Gravity } \\
\text { Onty }\end{array}$}} & \multirow{3}{*}{$\begin{array}{l}\text { Pounds } \\
\text { pet } \\
\text { Cubite } \\
\text { Foot: }\end{array}$} & \multirow{3}{*}{ Volume } & \multirow{3}{*}{$\begin{array}{l}\text { Weight } \\
\text { (tons) of } \\
1 \mathrm{MBF} \text { at } \\
\mathrm{MC} 20,00\end{array}$} & \multicolumn{5}{|c|}{ 1. $1=$ Good to $5=$ Poor } & \multirow{3}{*}{$\begin{array}{l}1-3 \\
\text { Ding } \\
\text { ing } \\
\text { Ense }\end{array}$} & \multirow{3}{*}{$\begin{array}{l}1 \\
\text { Mach } \\
\text { inabi- } \\
\text { Hiky }\end{array}$} & \multirow{3}{*}{$\begin{array}{c}106 \\
\text { Dutai } \\
\text { bit } \\
\text { ity. }\end{array}$} & \multirow{3}{*}{ 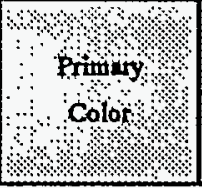 } & \multirow{2}{*}{\multicolumn{2}{|c|}{ 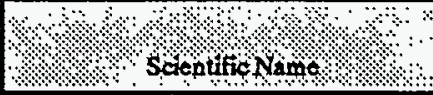 }} & \multirow[b]{3}{*}{$1+4$} & \multirow{3}{*}{. } \\
\hline & & & & & & \multirow{2}{*}{$\begin{array}{c}\text { Bend } \\
\text { ins } \\
\text { Strog }\end{array}$} & \multirow{2}{*}{$\begin{array}{l}\text { Comin } \\
\text { prest } \\
\text { sion }\end{array}$} & \multirow{2}{*}{ Hard } & \multirow{2}{*}{$\begin{array}{c}\text { Stiff. } \\
\text { ness. } \\
\text { :. } \\
\text { : }\end{array}$} & \multirow{2}{*}{\begin{tabular}{|l|} 
Shock \\
Rests \\
Hapce \\
\end{tabular}} & & & & & & & & \\
\hline & $\begin{array}{l}\text { Green } \\
\mathrm{yol}\end{array}$ & $\begin{array}{c}\text { Seg } \\
\text { soned }\end{array}$ & & & & & & & & & & & & & $6 \%$ & & & \\
\hline Hornbeam, American & 0.58 & 0.65 & 40.54 & 19.1 & $4,053.8$ & 4 & 4 & 2 & 4 & 1 & 1 & 2 & 6 & White & Carpinus & caroliniana & Hardwood & N. America \\
\hline Hornbeam, Europèan & 0.66 & 0.75 & 46.80 & & $4,680.4$ & 4 & 4 & 2 & 4 & 1 & 1 & 3 & 6 & White & Carpinus & betulus & Hardwood & Europe \\
\hline Horse-chestnut & 0.45 & 0.51 & 31.82 & & $3,182.7$ & 5 & 5 & 5 & 4 & 5 & 1 & 1 & 6 & Yellow White & Aesculus & hippocastanum & Hardwood & Europe \\
\hline ddigbo & 0.48 & 0.55 & 34.32 & 13.1 & $3,432.3$ & 4 & 3 & 3 & 3 & 4 & 1 & 1 & 2 & L Yel Brown & Texnduta & worenis & Hardwood & West Africa \\
\hline Iroko: & 0.56 & 0.64 & 39,94 & & 3,9939 & 3 & 2 & 1 & $z$ & & 1 & 2 & $\ddot{2}$ & Bpown $\$$ & Chloroptions & escetst & Hardwoo & Arnct \\
\hline Irotibaty & 0.99 & 1.12 & 69.89 & & $6,989,4$ & 1 & 1 & 1 & 1 & & 3 & 3 & 2 & Redspown & Euctyptis & $\mathrm{ppp}_{\mathrm{p}}$ & Hardwood & E Austritis \\
\hline Ironwood & 0.63 & 0.71 & 44.03 & 19.4 & $4,403.3$ & 2 & 3 & 2 & 3 & 1 & 1 & 2 & 6 & White & Ostrya & virginiana & Hardwood & N. America \\
\hline Jarrah & 0.67 & 0.82 & 51.17 & & $5,117.2$ & 2 & 1 & 1 & 2 & & 2 & 2 & 3 & Red Brown & Eucalyptus & marginata & Hardwood & W. Australia \\
\hline Jelutong & 0.36 & 0.40 & 25.16 & & $2,516.2$ & 5 & 3 & 5 & 3 & & 1 & 1 & 5 & Straw & Dyera & costulata & Hardwood & S. E. Asia \\
\hline Kupur & 0.04 & 0,72 & 4493 & & 44932 & 1 & 1 & 2 & 1 & & 2 & 4 & 2 & Red Brovo & Doyobalanops & $s p p_{n}$ & Elardwood & $80 \mathrm{ss}$ \\
\hline Kanh & 0.70 & 0.90 & 56.16 & & $5,616,4$ & 2 & $i$ & 1 & 1 & & 3 & 3 & 4 & Red brown & Encahplus & druenioolor & Rardwood & Woutralia \\
\hline knuri New zelating & 0.47 & $0.53:$ & 32.85 & & 3,2850 & 4 & $s$ & 4 & $3:$ & 3 & $i$ & 1 & 3 & LYel Brown & Asathit & austriblis & Sotwood: & Hew zealand \\
\hline Kempas & 0.77 & 0.87 & 54.29 & & $5,429.2$ & 2 & 1 & 1 & & & 1 & 3 & 4 & Red Brown & Koompassia & malaccensis & Hardwood & S. E. Asia \\
\hline Keruing & 0.67 & 0.75 & 46.82 & & $4,682.9$ & 1 & 1 & 2 & 1 & & 2 & 4 & 3 & Red Brown & Dipterocarpus & spp. & Hardwood & S. E. Asia \\
\hline Kingwood & 1.06 & 1.20 & 74.88 & & $7,488.6$ & & 1 & +1 & 1 & 1 & 2 & 2 & 2 & D. Brown Stp. & Dalbergia & cearensis & Hardwood & S. America \\
\hline $\mathrm{Kok}, \mathrm{l}$ & 056 & 064 & 39.94 & & 3,9939 & 2 & 2 & 2 & 2 & & 3 & 2 & 3 & park Brom & oponts & lebbok: & Hardpood & 19. \\
\hline Laburnum & 0.74 & 0.81 & $\$ 242$ & 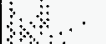 & $5,242.0:$ & & & 2 & $\because$ & & & 1 & 3 & DarkBror & Hownon & dasgyroddet & Hardwod & Eutop \\
\hline Larch Egstem 1 & 0.49 & 0.55 & 34.25 & 13.6 & $3,424.8$ & 3 & 3 & 5 & 3 & 4 & 1 & 3 & 4 & L Rod Bron & $10 x$ & 10 rivo & Softwood & Nonimerica \\
\hline Larch, European & 0.33 & 0.69 & 43.06 & & $4,305.9$ & 2 & 3 & 4 & 2 & & 2 & 2 & 4 & L. Red Brown & Larix & decidua & Softwood & Europe \\
\hline Larch, Siberian & 0.61 & 0.69 & 43.06 & & $4,305.9$ & 2 & 3 & 4 & 2 & & 2 & 2 & 4 & L. Red Brown & Larix & sibirica & Softwood & Europe/Asia \\
\hline Larch, Western & 0.48 & 0.54 & 33.55 & 13.2 & $3,354.9$. & 2 & 2 & 4 & 1 & 4 & 1 & 2 & 4 & L. Red Brown & Larix & occidentalis & Softwood & N. America \\
\hline táté, ludian & 0.75 & 0.85 & 53,04 & & $5,304,4$ & 2 & $1:$ & $1 \%$ & 1 & & 3 & $3:$ & 2 & Browit. & Tertutuitia & tonentoss & Hardwood & Indur \\
\hline tignumvitae & 0,98 & 123 & 76.75 & & $7,675.8$ & 18 & 1 & +1 & & & 3 & 3 & 1 & Oreep Brow & Quaiacum & stuctuin & Alatwood: & Strinca \\
\hline $\mathrm{LnO}$ & 048 & 0.55 & 34.32 & \% & 3,4323 & 4 & 4 & 5 & 3 & \% & 14 & 2 & 6 & YellowWhile & Tilf & vitsars & Harowod & Eurppto \\
\hline Locust, Black & 0.66 & 0.74 & 46.13 & 10.2 & $4,613.0$ & 1 & 1 & 1 & 1 & 3 & 1 & 2 & 1 & Dark Brown & Robinia & pseudoacacia & Hardwood & N. America \\
\hline Louro, Red & 0.55 & 0.62 & 38.69 & & $3,869.1$ & 2 & 2 & 3 & 3 & & 2 & 2 & 2 & Red Brown & Ocotea & rubra & Hardwood & S. America \\
\hline Lauan, Bagtikan & 0.48 & 0.54 & 33.55 & & $3,354.9$ & 2 & 2 & 2 & 2 & & 1 & 1 & 4 & L. Yel. Brown & Parashorea & plicata & Hardwood & S. E Asia \\
\hline Lauan, Dork Red & 0.44 & 0.49 & 30.75 & ४४ & 3,0753 & 3 & 2 & 4 & 2 & & 1 & 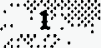 & 4 & Red Brow & Shorex & pegrosenis & Hardyod & Stosin \\
\hline Luns Light Red & 0.41 & 6.46 & 28.65 & 11.: & 2865.6 & 3 & 2 & 4 & 2 & & 1 & 1 & & Red Brown & Shorea & troo & Hardtood & $S, E, A r A$ \\
\hline tauan Mayap ss & 0,41 & 0,46 & 2865 & :at & 28656 & 3 & 3 & 3. & 1 & & 1 & $1 \%$ & 4 & L Red Brown & Shored & squpquas & Hardwood & S.EAstin \\
\hline Lauan, Red, Tanguile & 0.46 & 0.52 & 32.15 & 13.3 & $3,215.1$ & 2 & 2 & 4 & 1 & & 1 & 1 & 4 & Red Brown & Shorea & polysperma & Hardwood & S. E. Asia \\
\hline Lauan, White & 0.43 & 0.48 & 30.05 & 10.3 & $3,005.4$ & 3 & 2 & 4 & 2 & & 1 & 1 & 4 & Light Brown & Pentacme & contorta & Hardwood & S. E. Asia \\
\hline Magnolia, Cucumber & 0.44 & 0.49 & 30.75 & 13.6 & $3,075.3$ & 3 & 3 & 4 & 1 & 4 & 1 & 2 & 6 & L. Green Brown & Magnolia & acuminata & Hardwood & N. America \\
\hline Magnolio Southero & 0.46 & 052 & 32.15 & 123 & $3,215,1$ & 3 & 4 & 3 & 3 & 2 & 1 & 2 & 6 & Yoreen Brown & Magnolia & grandínora & Hardwood & NAnerica \\
\hline Mahogany & 0.45 & 050 & 31.45 & 79 & 3145,2 & 2 & $1:$ & 3 & 3 & 3 & 1 & 4 & 2 & $\mathrm{Rod} \mathrm{BNONO}$ & Swietenin & macophylla & Hardwood & S. Amera \\
\hline Mahogany, African & 0.478 & 0.53 & 33.07 & 9,2 & $3,307.5$ & 4 & 3 & 3 & 2 & 4 & 1 & 1 & 3 & Red Brown : & Khtaya & $\mathrm{spp}+\mathrm{Q}$ & Hardwood & Africa \\
\hline
\end{tabular}


Table 2. (continued) Properties of the World's Woods

\begin{tabular}{|c|c|c|c|c|c|c|c|c|c|c|c|c|c|c|c|c|c|c|}
\hline \multirow{3}{*}{ 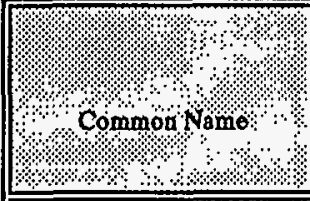 } & \multirow{2}{*}{\multicolumn{2}{|c|}{ 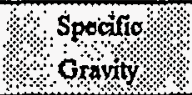 }} & \multirow{3}{*}{$\begin{array}{l}\text { Pounds } \\
\text { por } \\
\text { cibor } \\
\text { poof }\end{array}$} & \multirow{3}{*}{$\begin{array}{c}\text { ga } \\
\text { yolume } \\
\text { stirink } \\
\text { age }\end{array}$} & \multirow{3}{*}{ 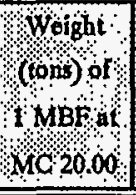 } & \multicolumn{5}{|c|}{ 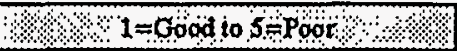 } & \multirow{3}{*}{$\frac{1-3}{\text { Ding }}$} & \multirow{3}{*}{ 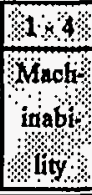 } & \multirow{3}{*}{$\begin{array}{l}180 \\
\text { pinis } \\
\text { bif } \\
\text { ity }\end{array}$} & \multirow{3}{*}{ 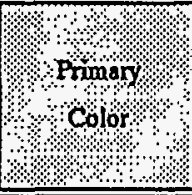 } & \multirow{2}{*}{\multicolumn{2}{|c|}{ 1. 1 Scontinc Namo }} & \multirow{3}{*}{ कै } & \multirow{3}{*}{ 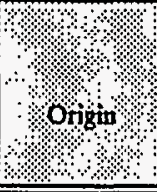 } \\
\hline & & & & & & \multirow{2}{*}{$\begin{array}{l}\text { ofod } \\
\text { ing } \\
\text { strng }\end{array}$} & \multirow{2}{*}{$\begin{array}{l}\text { Comp } \\
\text { prest } \\
\text { soon }\end{array}$} & \multirow{2}{*}{$\begin{array}{r}\text { Hard } \\
\text { ness } \\
\end{array}$} & \multirow{2}{*}{ 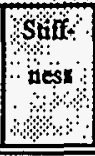 } & \multirow{2}{*}{$\begin{array}{l}\text { Shook } \\
\text { Resiss } \\
\text { conoo }\end{array}$} & & & & & & & & \\
\hline & Greet & sean & & & & & & & & & & & & & 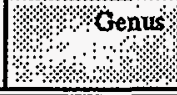 & \%ैpos & & \\
\hline Makore & 0.56 & 0.64 & 39.94 & 12.4 & $3,993.9$ & 3 & 2 & 2 & 2 & 3 & 2 & 4 & 2 & Red Brown & Tieghemella & heckelii & Hardwood & West Africa \\
\hline Maple, Bigleaf & 0.44 & 0.49 & 30.75 & 11.6 & $3,075.3$ & 3 & 3 & 3 & 3 & 5 & 2 & 2 & 6 & Light Brown & Acer & macrophyllum & Hardwood & N. America \\
\hline Maple, Black & 0.52 & 0.58 & 36.34 & 14.0 & $3,634,5$ & 2 & 3 & 2 & 2 & 3 & 2 & 1 & 6 & Light Brown & Acer & nigrum & Hardwood & N. America \\
\hline mplorposse & 0.58 & 066 & 41,18 & & $\% 187 \%$ & 3 & 2 & 2 & 2 & 3 & 2 & 2 & 8 & Aght Brown & Aobr & sp & Hariwod & 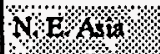 \\
\hline Maple Queousland & 0.48. & 0.55 & 3432 & ঋ & 4,4323 & 4 & 2 & 8 & 3 & 3 & 2 & 2 & 6 & brown & Findersin & braylyowa & Hardwood & Houstralia: \\
\hline$M a p l o, R e d-\curvearrowright$ & 0.49 & 0.55 & 34,25 & $13 \%$ & 3,4248 & 3 & 3 & 3 & 2 & 4 & 2 & 2 & 6 & L ght Brown & $A c \omega$ & rubrun & Handwood & $\mathrm{NA}_{\mathrm{Non}} \mathrm{ca}$ \\
\hline Maple, Silver & 0.44 & 0.49 & 30.75 & 12.0 & $3,075.3$ & 5 & 4 & 3 & 4 & 4 & 2 & 2 & 6 & Light Brown & Acer & saccharinum & Hardwood & N. America \\
\hline Maple, Sugar & 0.56 & 0.63 & 39.14 & 14.9 & $3,914.0$ & 2 & 2 & 2 & 1 & 3 & 2 & 1 & 6 & Light Brown & Acer & saccharum & Hardwood & N. America \\
\hline Masonia & 0.53 & 0.60 & 37.44 & & $3,744.3$ & 3 & 2 & 2 & & & 1 & 1 & 2 & Dark Brown & Masonia & altissima & Hardwood & West Africa \\
\hline Menghions & 800 & 0,08 & 4243 & १॰ & 4243,3 & \% & 3 & 2 & 3 & & 2 & 4 & 6 & $\mathrm{RedBrow}$ & Herotera & $s p p_{3}$ & Hardwood & 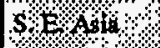 \\
\hline Mornil Dark Rod & 059 & 0,67 & 4181 & & $4181 \%$ & 2 & 2 & $3 \%$ & 2 & & 2 & 1 & 4 & Red Brown & Storeas: & pandiol & Handwood & SFAsin \\
\hline Merants Red & 0,4 & 048 & 30,05 & & 3,0054 & 2 & 2 & 4 & $i$ & & 1 & 1 & 4 & ARed Brolo & Shorea & ofoplitila & Hardwoon & SE ANA \\
\hline Meranti, Yellow & 0.55 & 0.62 & 38.44 & & $3,844.1$ & 2 & 2 & 3 & 2 & & 1 & 1 & 4 & Yellow Brown & Shorea & spp. & Hardwood & S.E. Asia \\
\hline Merbau & 0.70 & 0.80 & 49.92 & & $4,992.4$ & 3 & 2 & 3 & 2 & 3 & 1 & 3 & 1 & Red Brown & Intsia & spp. & Hardwood & $\begin{array}{l}\text { S. Asia/W. } \\
\text { Pacific }\end{array}$ \\
\hline Mersawa & 0.56 & 0.63 & 39.14 & & $3,914.0$ & 2 & 2 & 4 & 1 & & 1 & 4 & 4 & L Yel. Brown & Anisoptera & spp. & Hardwood & S. E. Asia \\
\hline Mota & 0.91 & 1.03 & 64,7 & + & 6427, & 2 & 1 & 1 & : & & 3 & 3 & 3 & Kor Browa & Mort & oxcelso & Hardwood & stritgat \\
\hline Muhinb & 079 & 0.90 & 5616 & +ै & 5,6164 & 3 & 2 & 2 & & & 2 & 3 & 4 & Red Btoph & 6\%ometh & plextant & Hard ood & Eost Arnot \\
\hline Munnge & 0,56 & 0,64 & 30.9 & खि & $3,4,9$ & 4 & 2 & & 2 & & 3 & 1 & 3 & Yoloy Brown & Pterocarpus & Angoltrist & Hardyou & Sonth frica \\
\hline Niangon & 0.56 & 0.64 & 39.94 & & $3,993.9$ & 2 & 1 & 2 & 3 & & 1 & 2 & 3 & Light Brown & Tarrietia & utilis & Hardwood & West Africa \\
\hline Oak, Black & 0.56 & 0.63 & 39.14 & 15.1 & $3,914.0$ & 2 & 3 & 2 & 3 & 3 & 3 & 1 & 5 & Light Brown & Quereus & velutina & Hardwood & N. America \\
\hline Oak, Bur & 0.58 & 0.65 & 40.54 & 12.7 & $4,053.8$ & 3 & 3 & 2 & 4 & 3 & 3 & 1 & 2 & Light Brown & Quercus & macrocarpa & Hardwood & N. America \\
\hline oul Chengbar & 0.61 & 0.68 & 4263 & (16) & 42035 & $z$ & 1 & 1 & 1 & 2 & 3 & 1 & $\$$ & Wh he Brown & ougcust & falcats $p^{2}$ & Hatowod & NAnterca \\
\hline 016 onestur & 0.57 & 0,64 & $30: 84$ & 164 & $3.9859:$ & 2 & 2 & 3 & 2 & 3 & 3 & 18 & 3 & Hobitsown & Ouerus: & pruniu & Hardwood & $\mathrm{NH}+\mathrm{mo}$ \\
\hline O4t, Dunmasi & 10.5 & 0.67 & 4181 & & 4,811 & 2 & 2 & 3 & 3 & 3 & 3 & 1 & 2 & Hght Brown & Querets & petpor & Hita hood & $\mathrm{Bu}+\mathrm{OP}$ \\
\hline Oak, English & 0.63 & 0.72 & 44.93 & & $4,493.2$ & 2 & 2 & 2 & 3 & 3 & 3 & 1 & 4 & Light Brown & Quercus & robur & Hardwood & Europe/Asia \\
\hline Oak, Japanese & 0.58 & 0.65 & 40.54 & & $4,053.8$ & 3 & 3 & 3 & 3 & 4 & 3 & 1 & 5 & Light Brown & Quercus & mongolica & Hardwood & N. E. Asia \\
\hline Oak, Laurel & 0.56 & 0.63 & 39.14 & 19.0 & $3,914,0$ & 2 & 3 & 2 & 2 & 3 & 3 & 1 & 5 & Light Brown & Quercus & laurifolia & Hardwood & N. America \\
\hline $0 \% 10 \%$ & 081 & 0,91 & 3061 & 147 & $5,661,4$ & \&: & 1 & 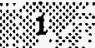 & 1 & 2 & 3 & 3 & 2 & Eught Brosho & Quercus & Yingintang & Harowood & $\mathrm{H}$-hnenca \\
\hline Oal, Nortbin Red & 156: & 0.63 & $\% 9.14$ & 137 & $3,90,0$ & 2 & 3 & 2 & 2 & 3 & 3 & 1 & 3 & Ugtit Brown & Quercus & rubtr? & Hardwood & N. America \\
\hline $04100+40$ & 057 & 0.64 & 3984 & 10.0 & 3,4839 & 2 & 3 & 2 & 3 & 3 & 3 & 1 & $2 \%$ & 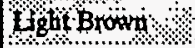 & Quercus & Hrata & Hardwood & $\mathrm{N} \mathrm{Anpt}_{\mathrm{S}}$ \\
\hline Oak, Persian & 0.59 & 0.66 & 41.23 & & $4,123,7$ & 2 & 2 & 2 & 3 & & 3 & 1 & & Light Brown & Quercus & castaneaefolia & Hardwood & W. Asia \\
\hline Oak, Pin & 0.58 & 0.65 & 40.54 & 14.5 & $4,053.8$ & 2 & 2 & 2 & 2 & 3 & 3 & 1 & 5 & Light Brown & Quercus & palustris & Hardwood & N. America \\
\hline Oak, Post & 0.60 & 0.67 & 41.93 & 16.2 & $4,193.6$ & 2 & 3 & 2 & 3 & 3 & 3 & 1 & 2 & Light Brown & Quercus & stellata & Hardwood & N. America \\
\hline Oak Red AYsos & 057 & 0.64 & 39,84 & 14.7 & 3,9839 & 2 & 3 & 2 & 2 & 3 & 3 & 1 & 5 & 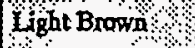 & Quirais & $8 x p$ & Hardwood & Namericas \\
\hline Oak Scarbet & 0.60 & 0.67 & 4193 & 147 & $4,193.6$ & 2 & 2 & $1 \%$ & 2 & 3 & 3 & 1 & 5 & Eight Brown & Querus: & oocoinea & Handwood & No Anterica \\
\hline
\end{tabular}




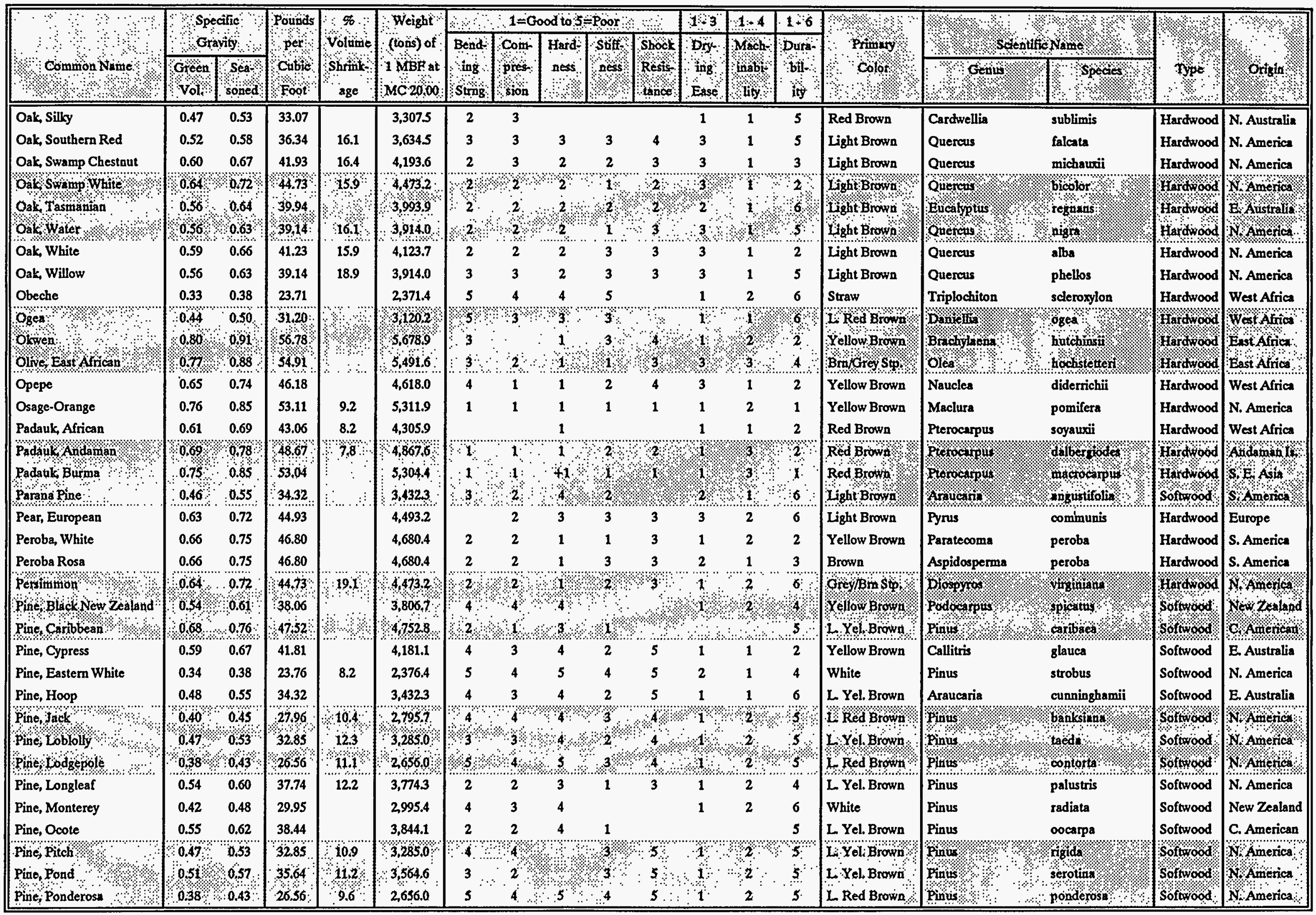




\section{¿ Table 2. (continued) Properties of the World's Woods}

\begin{tabular}{|c|c|c|c|c|c|c|c|c|c|c|c|c|c|c|c|c|c|c|}
\hline \multirow{3}{*}{$\because \%$} & \multirow{2}{*}{\multicolumn{2}{|c|}{ esporfo }} & \multirow{3}{*}{$\begin{array}{l}\text { Pounds } \\
\text { por } \\
\text { cubic } \\
\text { Foot }\end{array}$} & \multirow{3}{*}{$\begin{array}{c}\text { Wou } \\
\text { Stiringe } \\
\text { ange }\end{array}$} & \multirow{3}{*}{$\begin{array}{c}\text { Wright } \\
\text { (cons) of } \\
\text { 1 HBF at } \\
\text { MC 20.00 }\end{array}$} & \multicolumn{4}{|c|}{$1=$ Good to $S=$ Poor } & \multirow{3}{*}{\begin{tabular}{|c|} 
stiok \\
Resisis \\
$\vdots$ \\
tanco \\
\end{tabular}} & \multirow{3}{*}{$\begin{array}{l}1 \% 3 \\
\text { Drys } \\
\text { ing } \\
\text { Euse }\end{array}$} & \multirow{3}{*}{$\begin{array}{l}184 \\
\text { Mnow } \\
\text { inabi } \\
\text { lity }\end{array}$} & \multirow{3}{*}{$\begin{array}{l}106 \\
\text { Dura } \\
\text { bil } \\
\text { ity } \\
\end{array}$} & \multirow{3}{*}{ मे. Primary } & \multirow{2}{*}{\multicolumn{2}{|c|}{ W }} & \multirow{3}{*}{ 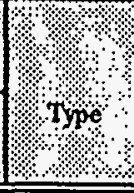 } & \multirow{3}{*}{ 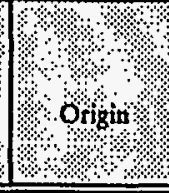 } \\
\hline & & & & & & \multirow{2}{*}{$\begin{array}{c}\text { Boid- } \\
\text { ing } \\
\text { Strng } \\
\end{array}$} & \multirow{2}{*}{$\begin{array}{l}\text { Com } \\
\text { pres: } \\
\text { sion } \\
\end{array}$} & \multirow{2}{*}{\begin{tabular}{l} 
Hurd \\
$\because \because$ ness \\
\hdashline
\end{tabular}} & \multirow{2}{*}{$\begin{array}{c}\text { Stiff: } \\
\text { niess } \\
\because\end{array}$} & & & & & & & & & \\
\hline & Greal & sofo: & & & & & & & & & & & & & ४.\%? & $8 . s$ & & \\
\hline Pine, Red & 0.41 & 0.46 & 28.65 & 11.5 & 65.6 & 5 & 4 & 5 & 3 & 4 & 1 & 2 & 5 & L. Red Brown & Pinus & resinosa & Softwood & rica \\
\hline Pine, Sand & 0.46 & 0.52 & 32.15 & 12.3 & 3.215 .1 & 3 & 3 & & 3 & & 1 & 2 & 5 & L. Yel. Brown & Pinus & clausa & Softwood & N. America \\
\hline Pine, Scotch & 0.42 & 0.48 & 29.95 & & $2,995.4$ & 4 & 3 & 4 & 3 & 4 & 1 & 2 & 5 & L. Red Brown & Pinus & sylvestris & Softwood & Europe \\
\hline Puteshotieh & 1046 & 0.52 & 3215 & 123 & $3,215.1$ & 3 & 3 & 4 & 2 & 4 & 1 & 2 & 5 & $141 B_{n} w$ & Ping & & soffood & $\mathrm{N}$ onth \\
\hline Pinoshas & 0,6 & 0.63 & 3914 & 12.2 & $3,914,0$ & 2 & 2 & 3 & 1 & 3 & 1 & 2 & 4 & LYel Brown & pinus: & elliottii & $\beta_{0}$ fipood & Nemerica \\
\hline pinesprue & 0.11 & 0.46 & 28.65 & 123 & $2,865.6$ & 4 & 4 & 4 & 3 & & 1 & 2 & 5 & Axol Bown & Pinus & labra & Sortwood & HAnrna \\
\hline Pine, Sugar & 0.35 & 0.39 & 24.46 & 7.9 & $2,446.3$ & 5 & 4 & 5 & 4 & 5 & 1 & 1 & 5 & White & Pinus & lambertiana & Softwood & erica \\
\hline Pine, & 0.45 & 0.50 & 31.45 & 11.9 & $3,145.2$ & 3 & 3 & 4 & 3 & 4 & 1 & 2 & 5 & L. Yel. Brown & Pinus & virgin & Softwood & N. America \\
\hline Pine, Western White & 0.36 & 0.40 & 25.16 & 11.8 & $2,516.2$ & 5 & 4 & 5 & 3 & 5 & 1 & 1 & 5 & White & Pinus & monticola & Softwood & erica \\
\hline How Who Now & 0.40 & 0,4 & 28.08 & & 38082 & 4 & 4 & & & & 1 & 1 & 6 & Whito & Podospus & dactyolod & Softwood & Hev zealand \\
\hline gatroe. & 0.56 & $0.6 \%$ & 39.98 & & 3093,9 & 4 & 4 & 4 & & 4 & & 2 & 8 & Gght Brown & Plataris & orientis & Hardorod & Etrope/Ast \\
\hline $800 \%$ & $0 \%$ & 051 & 3182 & . & 3,1827 & 4 & 3 & 3 & 3 & 4 & 8 & 1 & 6 & XYelorown & podoctpus & $8 \mathrm{PP}$ & Sortood & Fostarn: \\
\hline Purpleheart & 0.77 & 0.88 & 54.91 & & $5,491.6$ & 1 & 1 & +1 & 2 & 2 & 1 & 3 & 1 & Purple & Peltogyne & spp. & $\operatorname{cod}$ & rica \\
\hline Ramin & 0.59 & 0.66 & 41.23 & & $4,123.7$ & 2 & 1 & 3 & 1 & & 1 & 2 & 6 & White & Gonystylus & bancanus & Hardwood & S.E. Asia \\
\hline Raul & 0.48 & 0.55 & 34.32 & & 3,4323 & 2 & 4 & 3 & & & 3 & & 4 & L Red Brow & Nothofas & procero & Hatdwood & Somerics \\
\hline doedar eaterth & 044 & 049 & 30,75 & $78:$ & 3,0753 & 3 & 3 & 3 & 3 & 3 & 1 & 9 & 3 & $\mathrm{Re} / \%$ & Strotus & jífiflara & Solwoo & Homenca \\
\hline Redecdar Western & 0.31 & 0.35 & 2167 & 68 & 21667 & 3 & 4 & १\% & 4 & $\$$ & 2 & 2 & 2 & Pink Browa & Thuja: & plic & Sortood & ice \\
\hline Redwood, Old Growth & 0.38 & 0.43 & 26.56 & 6.8 & $2,656.0$ & 3 & 2 & 4 & 3 & 5 & 2 & 2 & 2 & Red & Sequoia & sempe & Softwood & N. America \\
\hline Redwo & 0.30 & 0.34 & 20.97 & 7.0 & $2,096.8$ & 5 & 3 & 5 & 4 & 5 & 2 & 2 & 2 & Red & Sequoia & sempe & Softwood & :rica \\
\hline Rimu & 0.53 & 0.60 & 37.44 & & $3,744.3$ & 4 & 5 & 4 & & & 1 & 2 & 6 & Yellow Brown & Dacrydium & cupressinum & Soltwood & New Zealand \\
\hline Rosetrod & 0.75 & 0.84 & 5242 & & 5,2420 & & 1 & 1 & $3:$ & & 1 & 3 & 2 & Doum Brow & Dalberga & latifotin & Handwod & India \\
\hline Rosevood Hrizatio & 076 & 0.86 & 5366 & $\%$ & $5,366.8$ & & 1. & & 8 & 1 & 2 & 2 & 2 & Drk Brown & Dalbergin & nigra & Hardwood & Somerna \\
\hline $\mathrm{Sal} / \%$ & 0.74 & 0.84 & 242 & & $5,242,0$ & 1 & $i$ & +1 & 1 & & 3 & 3. & 1 i. & Red Brown: & Shores & robums & Hardwood & Tratas $:$ \\
\hline pele & 0.56 & 0.64 & 39.94 & 13.8 & $3,993.9$ & 3 & 2 & 2 & 2 & 3 & 3 & 2 & 4 & Red Brown & Entandrophragma & cylindricum & Jardwood & Africa \\
\hline Sassafras & 0.42 & 0.47 & 29.35 & 10.3 & $2,935.5$ & 4 & 4 & 4 & 4 & 3 & 1 & 2 & 2 & rown & & & $\operatorname{cod}$ & N. America \\
\hline Satinwood, East Indian & 0.86 & 0.98 & 61.15 & 14.7 & $6,115.7$ & 1 & 1 & +1 & 1 & 2 & 2 & 3 & 1 & Golden Brown & Chloroxylon & swic & rood & India \\
\hline Saluwoodsonted & $0 . \$ 5$ & 8.63 & 30,0 & & 3.9315 & 2 & 3 & 3 & 2 & & 1 & t & 6 & OpinkBown & Cefapolfum & peralium & Hordwood & EAHshan \\
\hline Sourwood & 0.50 & 0.56 & 34.94 & 15.2 & $3,494.7$ & 3 & 3 & 3 & 2 & 3 & 1 & 2 & 6 & Light Brown & Oxydendrum & arboreum & wood & N. America \\
\hline Spanish Cedar & 0.34 & 0.38 & 23.76 & & $2,376.4$ & 5 & 4 & 3 & 4 & & 2 & 1 & 2 & Red Brown & Cedrela & odorata & rood & C. A \\
\hline ruoe, Black & 0.38 & 0.43 & 26.56 & 11.3. & $2,656.0$ & 5 & 4 & 5 & 3 & 5 & 1 & 2 & 6 & White & Picea & mariana & Softwood & N. America \\
\hline Huonglemtun & 0.33 & 0.37 & 23.06 & 110 & $2,306.5$ & 3 & 4 & 5 & 3 & 5 & $1:$ & 2 & 6 & White & Poes: & engetintintil & softoood & NAAnétor \\
\hline rwo, Norway $\}$ & 037 & 0,42 & 26,21 & & 26210 & 5 & 4 & 8 & 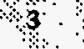 & 5 & 1 & 1 & 6 & White & Poes & bios & Solpood & Europe: \\
\hline noes Red & 0.36 & 0.40 & 25,16 & 129 & $2,516.2$ & 5 & 4 & $s$ & 3 & 3 & 1 & 2 & 6 & White & Pioes: & 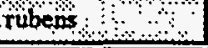 & 00 d & Ni America \\
\hline
\end{tabular}




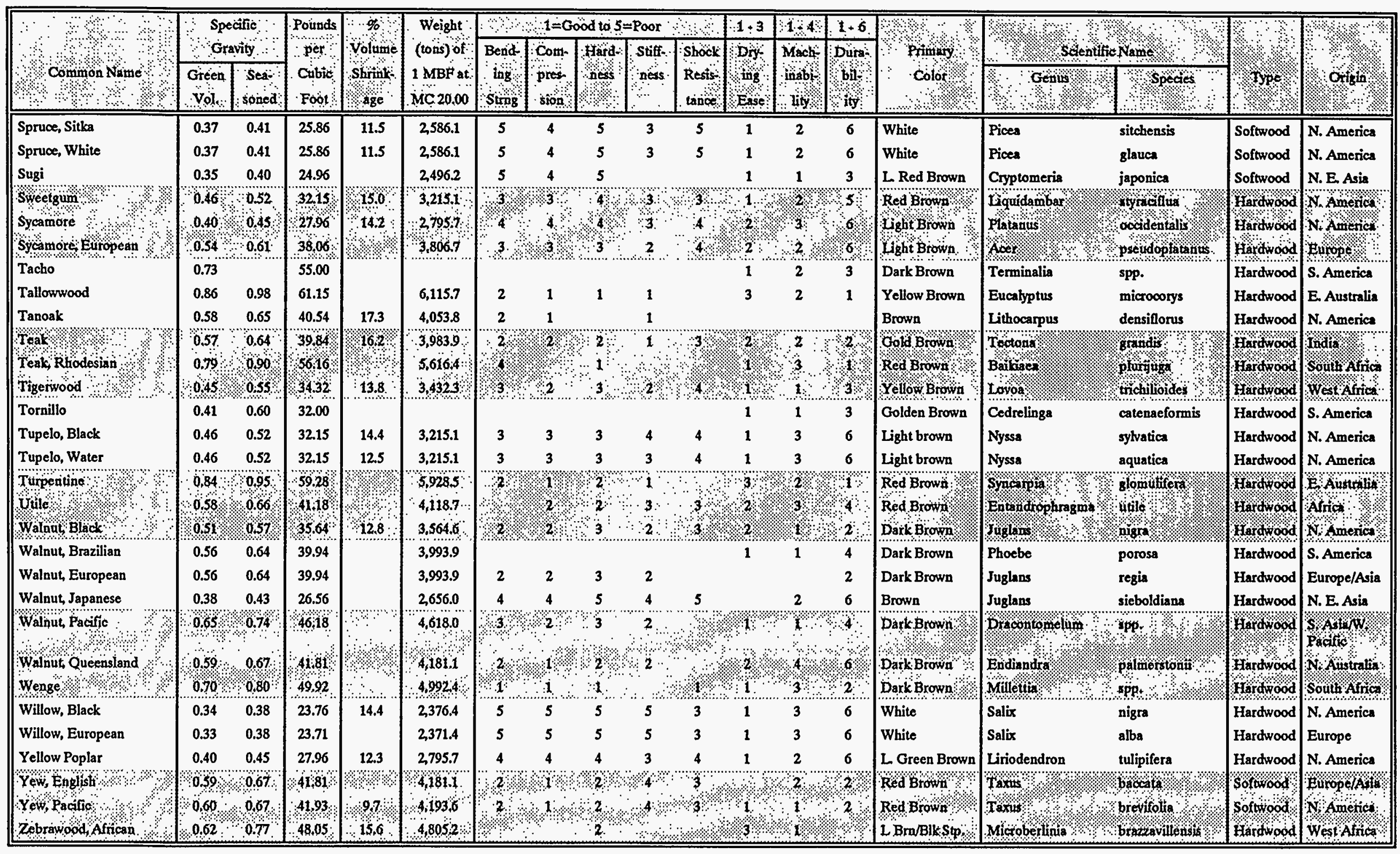




\section{Table 3.}

\section{Properties of Commonly}

\begin{tabular}{|c|c|c|c|c|c|c|c|c|c|c|c|c|c|c|c|}
\hline \multirow{5}{*}{ 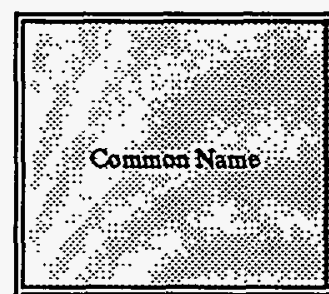 } & \multirow{4}{*}{\multicolumn{2}{|c|}{ 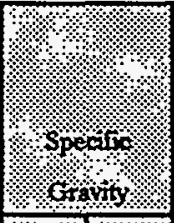 }} & \multirow{4}{*}{ (1. } & \multirow{5}{*}{$\int$} & \multirow{4}{*}{ 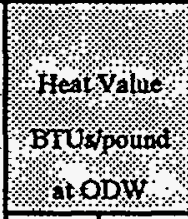 } & \multirow{5}{*}{ 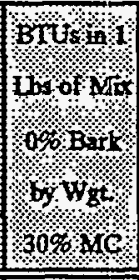 } & \multirow{4}{*}{\multicolumn{3}{|c|}{ 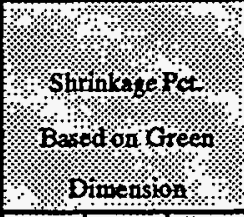 }} & \multicolumn{2}{|c|}{ S Sirsobint } & \multirow{5}{*}{ 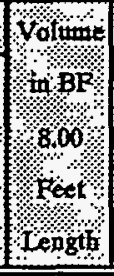 } & \multirow{5}{*}{$\begin{array}{l}\text { Weight } \\
\text { inibs: } \\
\text { : } \\
\\
\end{array}$} & \multirow{5}{*}{ 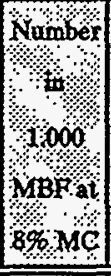 } & \multirow{5}{*}{$\begin{array}{l}\text { Weight of } \\
1,000 \\
\text { MBr at } \\
20 \% \text {. } \\
\text { Mc }\end{array}$} \\
\hline & & & & & & & & & & \multirow{4}{*}{ 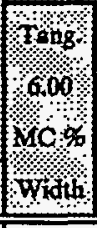 } & \multirow{4}{*}{ 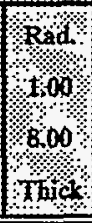 } & & & & \\
\hline & & & & & & & & & & & & & & & \\
\hline & & & & & & & & & & & & & & & \\
\hline & Gres: & 128 & oreen $12 \%$ & & Wood $/$ Bart & & 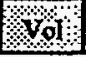 & Tang & Rad & & & & & & \\
\hline r. Red & 0.37 & 0.41 & $46.2 \quad 28.7$ & 100 & $8,000 \quad 8,410$ & 4,522 & 12.6 & 73 & 4.4 & 5.68 & 0.97 & 3.66 & 7.6 & 73 & 309.0 \\
\hline h, Black & 0.45 & 0.49 & \begin{tabular}{|ll}
51.9 & 34.2
\end{tabular} & 85 & $9,630 \quad 8,453$ & 5,776 & 15.2 & 7.8 & 5.0 & 5.66 & 0.96 & 3.63 & 9.2 & 275 & 808.2 \\
\hline Blue & 0.53 & 0.58 & $523 \quad 40.5$ & 58 & $9,630 \quad 8,453$ & 5,776 & 11.7 & 6.5 & 3.9 & 5.71 & 0.97 & 3.70 & 11.0 & 270 & 3,3075 \\
\hline & 63 & 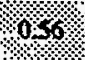 & $123 \cdot 3=397$ & 8. & $9630 \% 1 \times 453$ & 8,778 & $1 \%$ & 81 & 4.6 & 3.69 & $097 \%$ & 366 & 10.9 & 273 & 3,3075 \\
\hline & 1050 & 0.35 & 149.3 .1 .38 .4 & s8. & 96600.6453 & 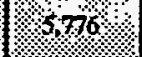 & 13.7 & 8. & 9.1 & 3.64 & $0.97 \%$ & 3.65 & 10.2 & 274 & 3,202 \\
\hline & 0.55 & 1060 & $48.0 \cdot 41, \%$ & 40 & $9,630 \% 8453$ & 5,776 & 134 & 7.8 & 8.8. & 8,66 & 0.96 & 3.64 & 112 & 275 & 3323 \\
\hline ooth & 0.36 & 0.39 & $44.3 \quad 27.3$ & 97 & $8,000 \quad 8,712$ & 4,522 & & 7.9 & 3.3 & 5.65 & 0.98 & 3.68 & 7.4 & 272 & 246.6 \\
\hline taking & 0.35 & 0.38 & $43.0 \quad 26.6$ & 97 & $8,000 \quad 8,712$ & 4,522 & & 6.7 & 3.5 & 5.71 & 0.97 & 3.71 & 7.3 & 270 & $2,184.2$ \\
\hline merican & 0.32 & 0.37 & $41.9 \quad 25.9$ & 110 & 8,000 & 4,522 & 15.8 & 9.3 & 6.6 & 5.59 & 0.95 & 3.55 & 6.4 & 282 & $1,997.0$ \\
\hline & 032 & 0.37 & $40.9 \% 25.9$ & 005 & 8,000 & 22 & 158 & 93 & 6.6 & S39: & $0.95 \%$ & 355 & 64: & 282 & 1997.0 \\
\hline ech, Amerted & 0.56 & $0.61 \%$ & $54,2,447$ & 53 & $8,600 \quad 7,993$ & 4.983 & 163 & $11: 0$ & 81 & $5 \$ 2$ & 0.96 & 354 & tof & 883 & $3,494.2$ \\
\hline$c h$ & 0.48 & 1055. & $S_{2} 4 \times 38$ & 15 & $0,340 \% 930 \%$ & $5,5,52$ & 162 & $8 \%$ & 63 & 3.62 & 0.95 & 357 & 36 & 280 & 2995.4 \\
\hline Ch, oweet & 0.60 & 0.65 & $62.2 \quad 45.4$ & 66 & $9,340 \quad 9,300$ & 5,552 & 15.6 & 9.0 & 6.5 & 5.60 & 0.95 & 3.56 & 20 & 281 & $3,744.3$ \\
\hline Ulow & 0.55 & 0.62 & \begin{tabular}{|ll}
57.0 & 43.3
\end{tabular} & 66 & $9,340 \quad 9,300$ & 5,552 & 16.7 & 9.2 & 7.2 & 5.60 & 0.95 & 3.53 & 10.9 & 283 & $3,432.3$ \\
\hline 10 & 0.33 & 0.36 & $49.0 \quad 25.2$ & 138 & 8,000 & 4,522 & 12.5 & 8.1 & 3.6 & 5.64 & 0.97 & 3.66 & 6.8 & 273 & 2059.4 \\
\hline itter? & 036 & 0.38 & 464 & 105: & 8,000 & 4,32 & 10.6 & 6.4 & 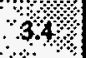 & 572 & 0.98 & 3,2 & 75 & 269 & 2246.6 \\
\hline forth & 0.38 & 0.41 & $410 \% 287$ & 73 & 8,000 & 43522 & 173 & 4.9 & $2: 5$ & 878 & 0.98 & 3,79 & 81 & 264 & $2,371.4$ \\
\hline & 047 & 0.50 & $45.2,449$ & 54 & & sod & 115 & 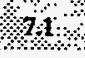 & $\therefore \quad>$ & 5.69. & 0.97 & 3.69 & 97 & 271 & 490.0 \\
\hline an & 0.40 & 0.43 & $\begin{array}{ll}54.9 & 30.1\end{array}$ & 120 & 8,600 & 4,983 & 11.6 & 6.7 & 3.4 & 5.71 & 0.98 & 3.71 & 8.3 & 270 & $2,496.2$ \\
\hline d, Balsam & 0.31 & 0.34 & $48.4 \quad 23.8$ & 150 & $\begin{array}{ll}8,800 & 8,422\end{array}$ & 5,137 & $\mid 10.5$ & 7.1 & 3.0 & 5.69 & 0.98 & 3.71 & 6.5 & 270 & 1,934.6 \\
\hline 4 Dac & 0.31 & 0.35 & $\begin{array}{ll}48.4 & 24.5\end{array}$ & 150 & $\begin{array}{ll}8,800 & 8,422\end{array}$ & 5,137 & 12.4 & 8.6 & 3.6 & 5.62 & 0.97 & 3.6 & 6.4 & 274 & 1,934.6 \\
\hline Easte & 0037 & $040 \%$ & 49.2280 & 413 & $8,800 \quad 8,422$ & $5: 137$ & 141. & 9.2 & 39 & 5.60 & 0.97 & 3.62 & 25 & 276 & 2309.0 \\
\hline Towerin & 0.64 & 003 & $64.3 .510 \%$ & 61 & $1+360$ & 5,368 & 20.8 & 118 & 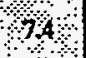 & 3.48 & 0,95 & 3.46 & $12 \%$ & 289 & $3,993.9$ \\
\hline 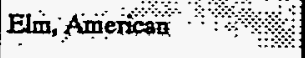 & $044 \%$ & 0.50 & 1840 & $88 \%$ & $8600 \quad 7 ; 385$ & 1,989 & 4.6 & 9.5 & 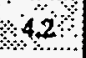 & 5,58 & 0.97 & tol & 93 & $2 \pi$ & $2,870.6$ \\
\hline$n, 1$ & 0.57 & 0.63 & $\begin{array}{|ll|}53.0 & 44.0\end{array}$ & 49 & 8,600 & 4,983 & 14.1 & 8.1 & 4.8 & 5.64 & 0.96 & 3.63 & 11.6 & 275 &, 557.1 \\
\hline 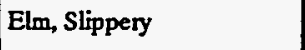 & 0.48 & 0.53 & $\begin{array}{ll}56.0 & 37.0\end{array}$ & 87 & $8,600 \quad 7,600$ & 4,983 & 13.8 & 8.9 & 4.9 & 5.61 & 0.96 & 3.60 & 9.7 & 277 & 995.4 \\
\hline & 0.49 & 0.53 & 50.1 & 64. & 8,600 & 4,983 & 16.9 & 8.9 & 4.8 & 5.61 & 0.96 & 3.61 & 9.9 & 27 & $\begin{array}{l}3,057.8 \\
\cdots\end{array}$ \\
\hline thong & 0.60. & 0.66 & $625 \%, 611$ & 67 & $9360 \quad 8423$ & $\$ 568$ & 13.6 & 8.5 & 19 & 561 & $0.0 \%$ & 3.60 & 12 & $2 \pi$ & 43 \\
\hline 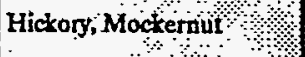 & 0.04 & $072:$ & $643 \% 50.3 \%$ & 6 & 9,360 & 8,568 & 178 & 110 & 7.7 & 5.52 & 0.94 & 34 & 125 & 288 & $3,993.9$ \\
\hline$x_{2}$ & 0.56 & 0.60 & 584,419 & 67 & $0,360,8,423$ & 85,56 & $136 \%$ & 8.9 & 49 & $5 \% 1$ & 0,06 & 3.60 & 113 & 277 & 3.49477 \\
\hline & 0.60 & 0.66 & $62.5 \quad 46.1$ & 67 & $9,360 \quad 8,423$ & 5,568 & 13.6 & 8.9 & 4.9 & 5.61 & 0.96 & 3.60 & 12.1 & 277 & 7443 \\
\hline & 0.66 & 0.75 & $65.9 \quad 52.4$ & 60 & $\begin{array}{ll}9,360 & 8,423\end{array}$ & 5,568 & 17.9 & 11.5 & 7.2 & 5.49 & 0.95 & 3.47 & 12.9 & 288 & 118.7 \\
\hline gbark & 0.64 & 0.72 & $63.9 \quad 50.3$ & 60 & $\begin{array}{ll}9,360 & 8,423\end{array}$ & 5,568 & 16.7 & 10.0 & 7.0 & 5.56 & 0.95 & 3.52 & 12.6 & 284 & $3,993.9$ \\
\hline & 1002. & 0.69 & $619 \%, 482$ & 60 & $9,3601.8423$ & 5,568 & $192 \%$ & 126 & 16 & 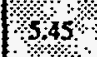 & 0.94 & $3: 43$ & $11 \%$ & 292 & $3,869,1$ \\
\hline Hickory, Water & $0.6 \%$ & 062 & 66.6 .133 & $\%$ & $3,360 \%$ & & 13.6 & 8.9 & $\%$ & 5,61 & 9.96 & 3.60 & 23 & 277 & $3,806.7$ \\
\hline 1by:American & 050. & 057 & 47.1 39.8 & 83 & 8,600 & 4,983 & 169 & 9.9 & 48 & 556 & 0.96 & $3.58 \%$ & 101 & 279 & 31202 \\
\hline & 0. & 0.67 & $61.0 \quad 46.8$ & 6 & 9,000 & 5,291 & 10.8 & 6.6 & 4.2 & 5.71 & 0.97 & 3.69 & 12.4 & 271 & ,744.3 \\
\hline & 0. & 0.70 & 52.8 & 46 & 8,800 & 5,137 & 19.1 & 11.4 & 5.7 & 5.50 & 0. & & 1.4 & 285 & 619.5 \\
\hline onwood & 0.63 & 0.70 & $60.1 \quad 48.9$ & 53 & 9,300 & 5,522 & 19.4 & 10.0 & 8.5 & 5.56 & 0.94 & 3.48 & 12.3 & 288 & $3,931.5$ \\
\hline
\end{tabular}




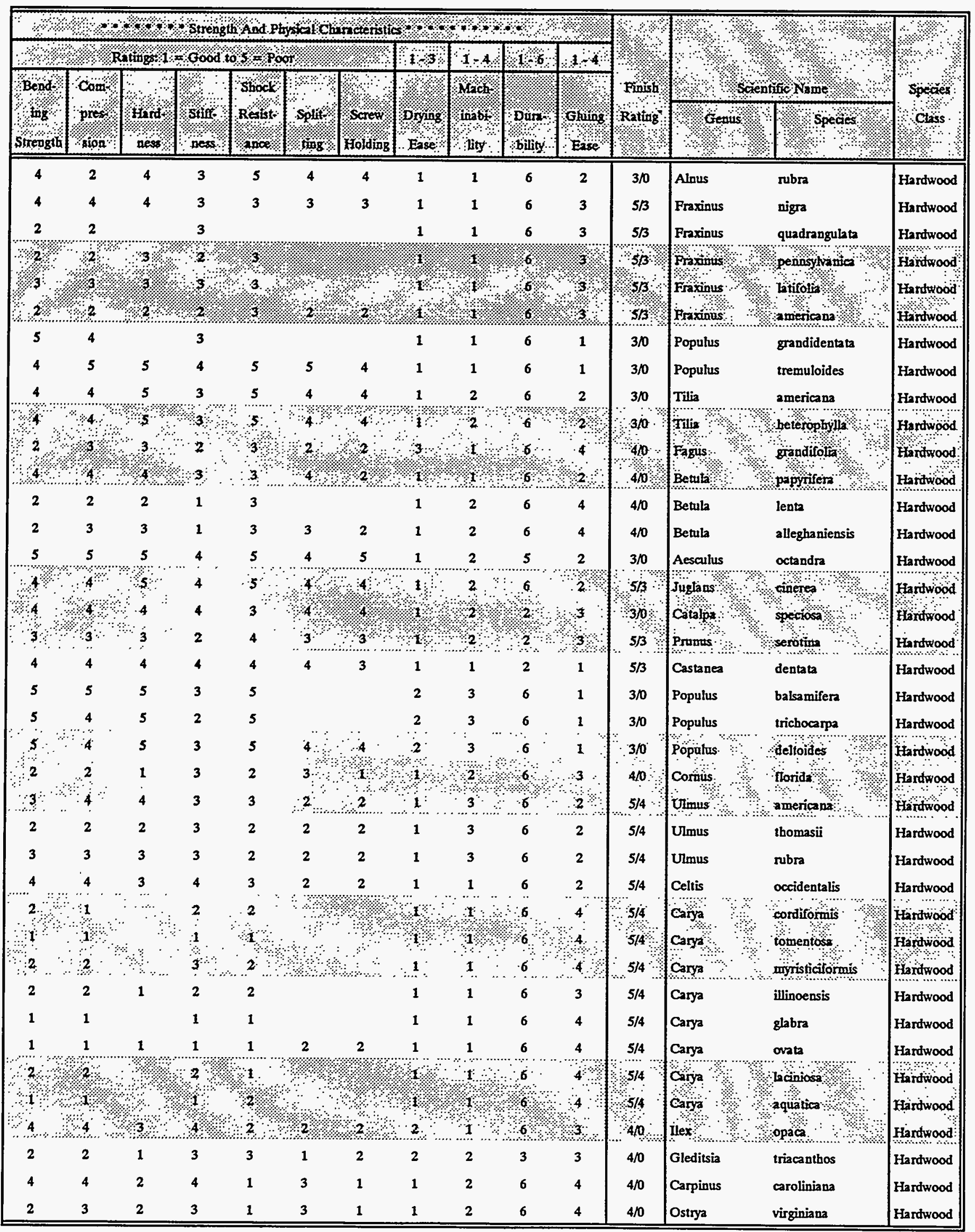




\begin{tabular}{|c|c|c|c|c|c|c|c|c|c|c|c|c|c|c|c|c|c|}
\hline \multirow{3}{*}{ 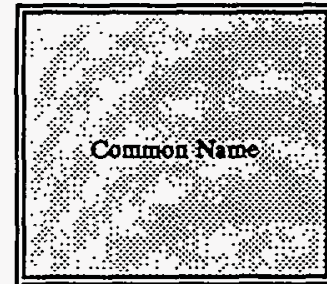 } & \multirow{2}{*}{\multicolumn{2}{|c|}{ 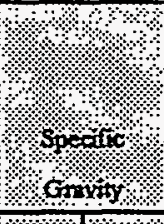 }} & \multirow{2}{*}{\multicolumn{2}{|c|}{ 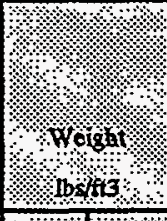 }} & \multirow{3}{*}{ Gren } & \multirow{2}{*}{\multicolumn{2}{|c|}{ 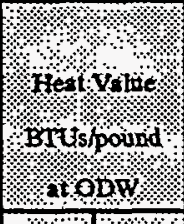 }} & \multirow{3}{*}{ 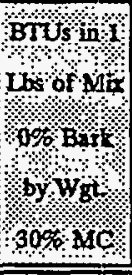 } & \multirow{2}{*}{\multicolumn{3}{|c|}{ 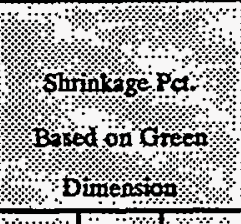 }} & \multicolumn{2}{|c|}{ Sta pin } & \multirow{3}{*}{ 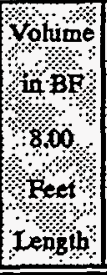 } & \multirow{3}{*}{ webbt } & \multirow{3}{*}{$\begin{array}{c}\text { Number } \\
\text { 1000 } \\
\text { HBP } \\
8 \% \mathrm{MC}\end{array}$} & \multirow{3}{*}{ 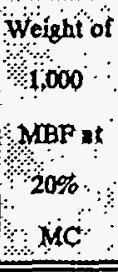 } \\
\hline & & & & & & & & & & & & \multirow{2}{*}{$\begin{array}{l}6.00 \\
\text { Me } \\
\text { with }\end{array}$} & \multirow{2}{*}{ Ridi } & & & & \\
\hline & Oreen & $12 \%$ & Greep & $12 \%$ & & wood & Bark & & rol & Tang & R,o & & & & & & \\
\hline caust, Blact & 0.66 & 0.69 & & 48.2 & 41 & 9,360 & & 5,568 & & & & 5.68 & 0.97 & 66 & 36 & 3 & \\
\hline 281 & 0.44 & 0.48 & 56.6 & 33.5 & 106 & 8,600 & & 4,983 & 13.6 & 8.8 & 5.2 & 5.61 & 0.96 & 3.60 & 8.9 & 278 & $2,745.8$ \\
\hline Magn & 0.46 & 0.50 & 59.1 & 34.9 & 106 & 8,600 & & 4,983 & 123 & 6.6 & 5.4 & 5.71 & 0.96 & 3.66 & 9.4 & 274 & 6 \\
\hline Mople, Bigleal & 0,4 & 048 & 45,0 & 33.5 & 64 & 8,40 & 8,293 & 4,829 & 116 & 71 & 3.7 & 569 & 0.97 & 369 & 91 & $2 \pi 1$ & $2,745.8$ \\
\hline Maple, & 0.52 & 037 & 519 & 308 & 60 & 8.400 & 8,230 & 4,829 & 140 & 93 & 48 & 539 & 0.96 & 3.60 & 105 & 278 & $3,25 x$ \\
\hline MapteqRed & $0 \%$ & 0.54 & 304 & 317 & 64 & $8+800$ & 8,29 & 4829 & 134 & 82 & 40 & 504 & 097 & 365 & 10,0 & $2+4$ & $3,057,8$ \\
\hline aph & 0.44 & 0.47 & 48.9 & 328 & 78 & 8,400 & 8,293 & 4,829 & 12.0 & 72 & 3.0 & 5.68 & 0.98 & 3.7 & 9.2 & 270 & .8 \\
\hline apl & 0.56 & 0.63 & 55.9 & 44.0 & 60 & 400 & 8,230 & 29 & 14.9 & 9.5 & 4.9 & 5.58 & 0.96 & 3.59 & 11.3 & 279 & .494.7 \\
\hline ak, & 0.56 & 0.61 & 60.5 & 426 & 73 & 9,360 & 8,896 & 5,568 & 15.1 & 11.1 & 4.4 & 5.51 & 0.97 & 3.56 . & 11.2 & 281 & \\
\hline 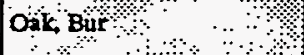 & 0.58 & 0,64 & 626 & 448 & 73 & $9510 \%$ & 745 & 5,683 & 121 & $8: 9$ & 44 & 561 & 0.97 & 362 & 118 & 276 & $3 ; 619.5$ \\
\hline oak & 0.6 & 0.68 & 678 & 475 & 78 & 9,360 & 8,896 & 5,568 & 161 & 113 & 47 & 5,50 & 0.97 & 3,54 & rat & 282 & 806.7 \\
\hline 6 & 605 & 0.66 & 615 & 46.1 & 83 & 9,510 & 7.450 & 5,683 & 16,4 & 108 & 3 & 52 & 0.96 & 354 & 1 & 282 & $3,557.1$ \\
\hline Dak. & 0.56 & 0.63 & 60.5 & 44.0 & 73 & 9,360 & 8.896 & 5,568 & 19.0 & 9.9 & 4.0 & 5.56 & 0.97 & 3.60 & 11.3 & 278 & $3,494.7$ \\
\hline Oak, Live & 0 & 0.89 & 75.8 & 62.2 & 50 & 10,000 & & 6,060 & 27. & 9. & 6. & 5 & 0.95 & 3.54 & 16.1 & 282 & 6.8 \\
\hline ak, & 0.56 & 0.63 & 60.5 & 44.0 & 73 & 9,360 & 8,896 & 5,568 & 13.7 & 8.6 & 4.0 & 5.62 & 0.97 & 3.64 & 11.4 & 275 & .7 \\
\hline$\therefore$ & 0.5 & 063 & 615 & 40 & 73 & 9,510 & 7,50 & 5 & 16,0 & 127 & 5.3 & 5,44 & 09 & 349 & 31,2 & $287:$ & $3,557.1$ \\
\hline Dak, pin: & 0.58 & 0.63 & 626 & 44,0 & 13 & 9360 & 8,896 & 5,568 & 14 & 95 & 43 & 5,58 & 0,9 & 360 & $n$ & 27 & $3,619,5$ \\
\hline Pak Post P & 606 & 0.67 & 648 & 468 & 13 & 9,510 & 7,450 & 5,683 & 162 & $98 \%$ & 5.4 & 5.57 & 0.96 & 357 & 120 & 280 & 7 \\
\hline Dak, & 0.57 & 0.62 & 63.3 & 43.3 & 78 & 9,360 & 8,896 & 5,568 & 14.7 & 8.9 & 4.2 & 5.61 & 0.97 & 3.62 & 11.6 & 276 & 3,557.1 \\
\hline 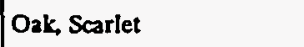 & 0.60 & 0.67 & 64.8 & 46.8 & 73 & 9,360 & 8,896 & 5,568 & 14.7 & 10.8 & 4.4 & 5.52 & 0.97 & 3.56 & 12.0 & 281 & - \\
\hline thern Red & 0.52 & 0.59 & 57.8 & 41.2 & 78 & 9,360 & $\begin{array}{l}8,896 \\
\ldots\end{array}$ & 5,568 & 16.1 & 11.3 & 4.7 & 5.50 & 0.97 & 3.54 & 103 & 282 & $3,245.1$ \\
\hline$\because$ & 0,60 & 0.67 & 64.8 & 46.8 & 73 & 9,510 & $\therefore$ & 5,683 & 16.4 & 10.8 & . & 552 & 096 & 354 & 11.9 & 282 & .3 \\
\hline$a k$ & 0.64 & 0.2 & 69.1 & 503 & 33 & 9,510 & 7450 & 5,683 & 15.9 & 92 & 53 & 560 & 0.96 & 3.59 & 129 & 279 & $3,993.9$ \\
\hline Oak, Water & 0,56 & 063 & 63,2 & 140 & 81 & 9360 & $0,0 \times 0$ & 5,568 & 16.1 & 98 & 44 & 5,57 & 0.97 & की, 59 & 113 & 278 & 194.7 \\
\hline 12 & 0.59 & 0.67 & 63.7 & 46.8 & 73 & 9,510 & 7.450 & 5,683 & 15.9 & 9.2 & 53 & 5.60 & 0.96 & 3.59 & 11.9 & 279 & 6.9 \\
\hline Oak, Willow & 0.56 & 0.69 & 61.9 & 48.2 & 77 & 9,360 & 8,896 & 5,568 & 18.9 & 9.6 & 5.0 & 5.58 & 0.96 & 3.58 & 11.3 & 279 & 4.7 \\
\hline Osage & 0.76 & 0.80 & 62.1 & 55.9 & 31 & 9,800 & & on & 9.2 & & & 6.00 & $\mathrm{~m}$ & 4.00 & 17.1 & 250 & ond \\
\hline retsing & 0.64 & 0.74 & 631 & 517 & 38 & 9.400 & & 599 & 19.1 & iit & & 5.51 & 094 & 3.46 & 124 & 289 & $3,993.9$ \\
\hline Sassafins & 0.4 & 0.45 & 440 & 314 & 68 & 8,6 & & 4,983 & 10.3 & $6: 2$ & $40 \%$ & 5.73 & 0.97 & 371 & 8.7 & 270 & 621.0 \\
\hline Sotrwood & 030 & 0.55 & 53.0 & 38.4 & 00 & 8,600 & & 4,983 & 152 & 8.9 & 63. & 5.61 & 0.95 & 3.57 & 10.0 & 280 & $3,120.2$ \\
\hline 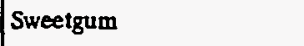 & 0.46 & 0.51 & 55.1 & 35.6 & 92 & 8,600 & 7,650 & 4,983 & 15.0 & 9.9 & 5.2 & 5.56 & 0.96 & 3.5 & 9.2 & 280 & 0.6 \\
\hline Sycamore & 0.40 & 0.49 & 51.9 & 34.2 & 108 & 8,600 & 7,877 & 4,983 & 14.2 & 7.6 & 5.1 & 5.67 & 0.96 & 3.64 & 8.2 & 275 & 196.2 \\
\hline . & 0.58 & & 61.5 & & 70 & 8,000 & & 4,522 & 17.3 & 11.7 & 4.9 & 5.4 & 0.96 & 3.5 & 11.5 & 284 & $3,619.5$ \\
\hline Tupelo, Black & 0 & 040 & $\$ 45$ & i. & $\sin$ & 8,600 & & भी & 14 & 8 & 5.18 & 5.62 & 096 & $3.60 \%$ & 93 & 277 & $2 ; 870.6$ \\
\hline Tupelo Water: & 0.46 & 050 & 66.0 & 49 & 130 & $8600^{\circ}$ & 8,412 & 4,983 & 125 & 76 & 42 & 5,67 & 0.97 & 3.66 & 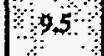 & 273 & 2070.6 \\
\hline Walnut, Blacks $\because \because$ & $0 \mathrm{si}$ & 0.55 & 57.9 & 38.4 & 82 & 8,600 & 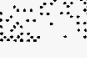 & 4,983 & 128 & 78 & 5. & 5.66 & 0.96 & $3.62 \%$ & 10.4 & 276 & 3.182 .7 \\
\hline Willow, Black & 0.34 & 0.37 & 50.7 & 25.9 & 139 & 8,000 & 7,648 & 4,522 & 14.4 & 8.1 & 2.6 & 5.64 & 0.98 & 3.69 & 7.0 & 271 & $2,121.8$ \\
\hline Yello & 0.40 & 0.42 & 46.2 & 29.4 & 85 & 9,630 & 8,956 & 5,776 & 12 & 7.1 & 4.0 & 5.69 & 0.97 & 3.68 & 8.3 & 272 & .2 \\
\hline Baldc & 0.42 & 0.46 & 51.1 & 32.1 & 95 & 10,660 & & 6,568 & 10.5 & 6.2 & 3.8 & 5.73 & 0.97 & 3.71 & 3.8 & 269 & $2,621.0$ \\
\hline
\end{tabular}




\begin{tabular}{|c|c|c|c|c|c|c|c|c|c|c|c|c|c|}
\hline \multicolumn{11}{|c|}{ 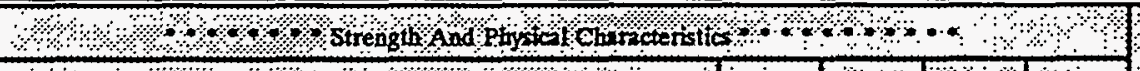 } & \multirow{5}{*}{ Ratinis } & \multirow{3}{*}{ (3) } & \multirow{5}{*}{$\mid \begin{array}{ll}0 \\
\text { speces } \\
\text { Class } \\
\text { c }\end{array}$} \\
\hline \multicolumn{7}{|c|}{ 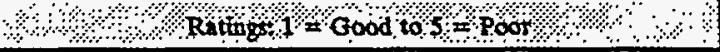 } & $1-3$ & 14. & 16 & $1+4$ & & & \\
\hline Send & oig & & & Shock & & & & Hach & + & & & & \\
\hline tos & prest. & Hata- & stiffif & Retist & sphtits & screti & Drying & insib: & purs & Ghing & & Gen & \\
\hline Strength & sions & ress & $\operatorname{sen}$ & anoes & tims & Holding & Ease & lity & bility. & Eise & & $\%$ & \\
\hline 1 & 1 & 1 & 1 & 3 & 2 & 1 & 1 & 2 & 1 & 4 & $4 / 0$ & pseudoac & Hardwood \\
\hline 3 & 3 & 4 & 1 & 4 & & & 1 & 2 & 6 & 2 & $3 / 0$ & acuminata & Hardwood \\
\hline 3 & 4 & 3 & 3 & 2 & 3 & 3 & 1 & 2 & 6 & 2 & 310 & grandiflora & Hardwood \\
\hline 3 & & 32 & 3 & 5 & & & 2 & 2 & 6 & 3 & 40 & macrophy & Hondivood \\
\hline 2 & & \% & & & 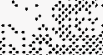 & & 2 & 稵 & 6 & 4 & 40 & Aoer & Ynandwood d \\
\hline 3 & & 3 & 2 & 4 & 3. & 2 & 2 & 2 & 6 & 3 & 40 & $A c 0 r$ & Hantwood \\
\hline 5 & 4 & 3 & 4 & 4 & & & 2 & 2 & 6 & 3 & $4 / 0$ & saccharinu & Hardwood \\
\hline 2 & 2 & 2 & 1 & 3 & 1 & 2 & 2 & 1 & 6 & 4 & $4 / 0$ & saccharum & Hardwood \\
\hline 2 & 3 & 2 & 3 & 3 & 2 . & 2 & 3 & 1 & 5 & 3 & $5 / 4$ & Quercus & Handwood \\
\hline & & 2 & 4 & 3 & 2 & 1 & 3 & & 2 & 3 & $:^{5 / 4}$ & Querais & Hartuood \\
\hline & & i & 1 & 2 & 2 & : & 3 & 1 & 5 & $\therefore$ & 544 & Querans $\%$ falgta va & Hardwood \\
\hline 2 & & 3 & 2 & 3 & 2 & is & 3 & $i$ & 2 & 3 & $5 / 4$ & Querais & Hardwood \\
\hline 2 & 3 & 2 & 2 & 3 & 2 & 2 & 3 & 1 & 5 & 3 & $5 / 4$ & burifolia & Handwood \\
\hline 1 & 1 & 1 & 1 & 2 & 1 & 1 & 3 & 3 & 2 & 4 & $5 / 4$ & virginjana & Handwood \\
\hline 2 & 3 & 2 & 2 & 3 & 2 & 2 & 3 & 1 & 5 & 3 & $5 / 4$ & Quercus & Hardwood \\
\hline & & 2 & 3 & 3 & 2 & $i$ & 3 & 1 & 2 & 3 & $5 / 4$ & Querais: & Fandivood: \\
\hline & & $2:$ & 2 & 3 & 2 & $\therefore$ & 3 & 1 & 5 & 3 & $5 / 4$ & Quercas & Haxdwood \\
\hline 2 & 3 & 2 & 3 & 3 & 2 & 1 & 3 & 4 & 2 & 3 & 514 & Querus & Hindwood \\
\hline 2 & 3 & 2 & 2 & 3 & 2 & 2 & 3 & 1 & 5 & 3 & $5 / 4$ & Quercus & Handwood \\
\hline 2 & 2 & 1 & 2 & 3 & 2 & 2 & 3 & 1 & 5 & 3 & $5 / 4$ & Queraus & Handwood \\
\hline 3 & 3 & 3 & 3 & 4 & 2 & 2 & 3 & 1 & 5 & 3 & $5 / 4$ & Quercus & Hardwood \\
\hline & & 2 & 2 & 3 & 2 & 1 & 3 & 1 & 3 & 3 & $5 / 4$ & Quercus $\quad$ michautii & Haxdwood \\
\hline 2 & $2:$ & 2 & 1: & 2 & 2 & 1 & 3 & 1 & 2 & 3 & $s / 4$ & Queraus biolor & Hardwood \\
\hline 2 & 2 & 2 & 1 & & 2 & 2 & 3 & 1 & 5 & 3 & $5 / 4$ & Quercus \}n & Hardwood \\
\hline 2 & 2 & 2 & 3 & 3 & 2 & 1 & 3 & 1 & 2 & 3 & $5 / 4$ & Quercus & Hardwood \\
\hline 3 & 3 & 2 & 3 & 3 & 2 & 2 & 3 & 1 & 5 & 3 & $5 / 4$ & Quercus & Handwood \\
\hline 1 & 1 & 1 & 1 & 1 & 1 & 1 & 1 & 2 & 1 & 4 & $4 / 0$ & pomifera & Handwood \\
\hline 2 & 2 & 1 & 2 & 34 & 2 & 4 & $\mathbf{1}$ & 2 & 6 & 4 & 40 & Diospyios $\%$ virgumana & Hardwood \\
\hline 4 & 4 & \% & 4 & :3: & 3 & 3 & 1 & 2 & $2 \%$ & 3 & 410 & Sassafras & Hardwood \\
\hline 3 & 3 & $\therefore$ & 2 & 3 & 2 & 2 & 1 & 2 & 6 & $3^{2}$ & 310 & Oxydendrum arboreum & Hardwood \\
\hline 3 & 3 & 4 & 3 & 3 & 3 & 3 & 1 & 2 & 5 & 2 & $3 \%$ & Liquidambar & Handwood \\
\hline 4 & 4 & 4 & 3 & 4 & 3 & 3 & 2 & 3 & 6 & 3 & $4 / 0$ & occidentalis & Hardwood \\
\hline 2 & 1 . & & 1. & & & & & & & & & Lithocarpus & Hardwood \\
\hline 3 & & 3 & 4 & 4 & 3 & 2 & 1 & 3 & 6 & 3 & 40 & Nissa & Handwood \\
\hline 3 & 3 & 3 & 3 & 4 & 3 & 2 & $1 \cdots$ & 3 & 66 & 3 & $4 / 0$ & Nyssa & Fiardwood \\
\hline 2 & 2 & $3 \%$ & 2 & 3 & 2 & 2 & 2 & 1 & 2 & 3. & $5 / 3$ & Juglans o atgra & Hindwood \\
\hline 5 & 5 & 5 & 5 & 3 & 4 & 4 & 1 & 3 & 6 & 1 & $3 / 0$ & Salix & Hardwood \\
\hline 4 & 4 & 4 & 3 & 4 & 4 & 4 & 1 & 2 & 6 & 1 & $3 / 0$ & Liriodendron & Handwood \\
\hline 4 & 3 & 5 & 3 & 4 & 4 & 3 & 1 & 2 & 3 & 1 & $1 / 0$ & distichum & Handwood \\
\hline
\end{tabular}




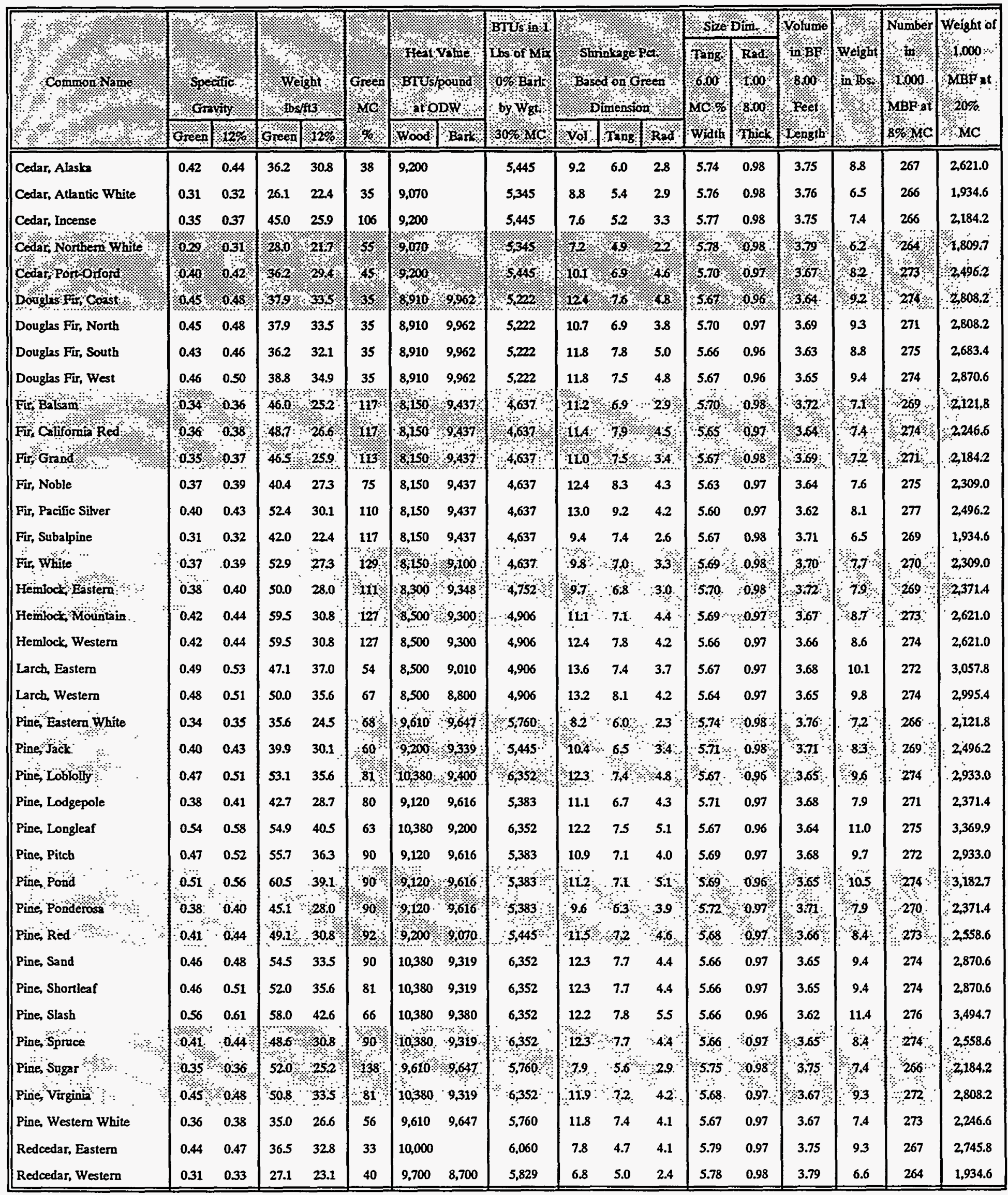




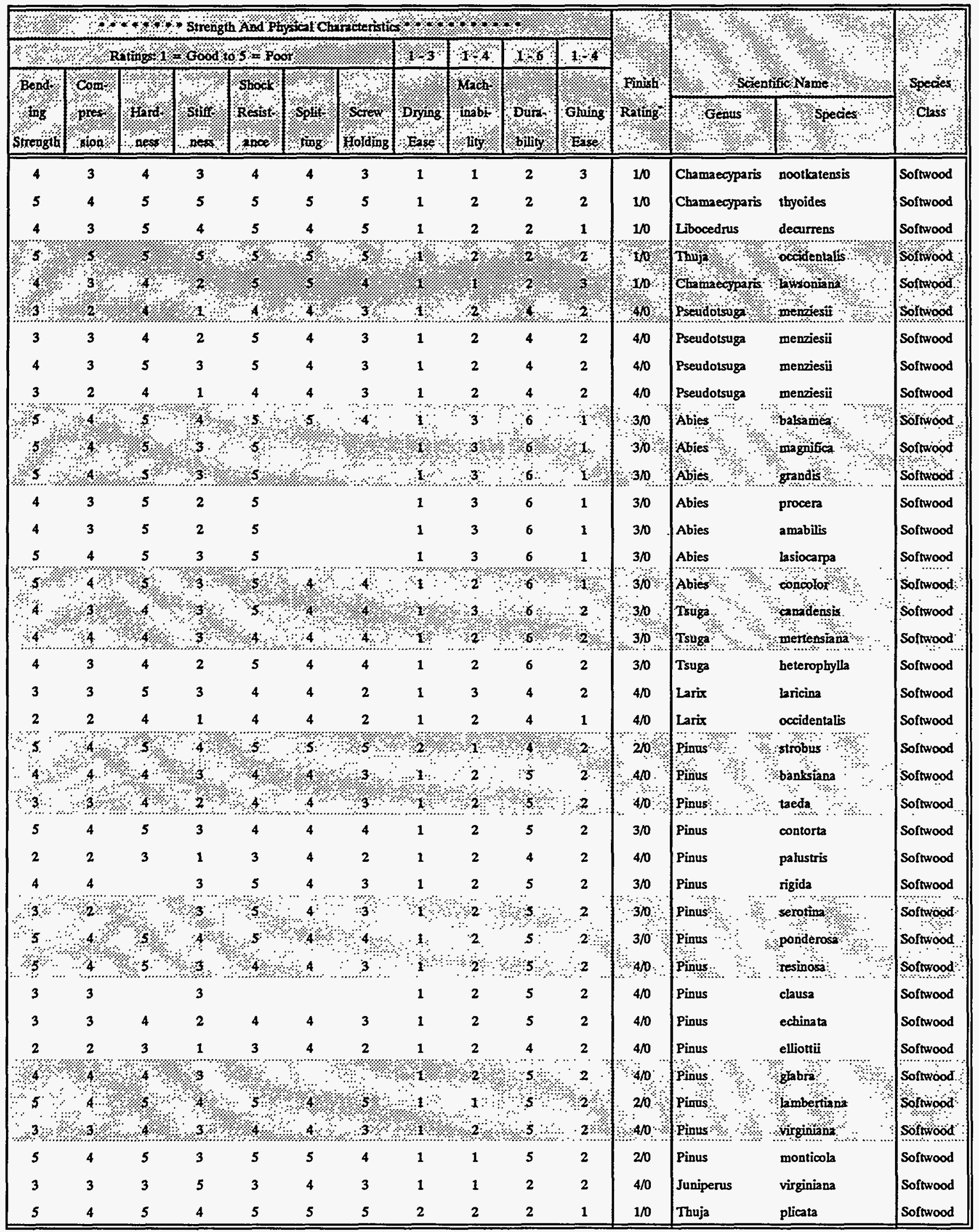




\begin{tabular}{|c|c|c|c|c|c|c|c|c|c|c|c|c|c|c|c|}
\hline \multirow{3}{*}{ (3) } & \multirow{2}{*}{\multicolumn{2}{|c|}{ 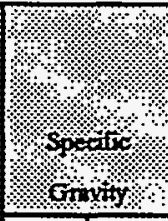 }} & \multirow{2}{*}{ 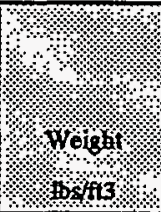 } & \multirow{3}{*}{ 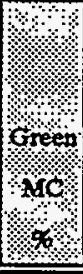 } & \multirow{2}{*}{ 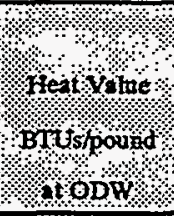 } & \multirow{3}{*}{ 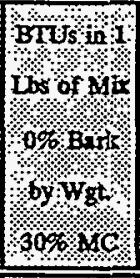 } & \multirow{2}{*}{\multicolumn{3}{|c|}{ \% }} & \multicolumn{2}{|c|}{ Ske plun } & \multirow{3}{*}{$\begin{array}{l}\text { olume } \\
\text { 10. BP } \\
8.00 \\
\text { Feet } \\
\text { Yength }\end{array}$} & \multirow{3}{*}{ 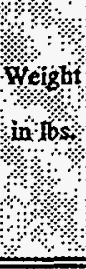 } & \multirow{3}{*}{ 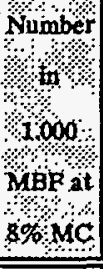 } & \multirow{3}{*}{$\begin{array}{l}\text { Weight ot } \\
1.000 \\
\text { MBp at } \\
\text { 20\% } \\
\text { MCC }\end{array}$} \\
\hline & & & & & & & & & & \multirow{2}{*}{ 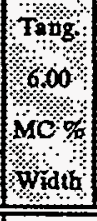 } & \multirow{2}{*}{$\begin{array}{l}\text { Rad } \\
1.00 \\
8.00 \\
\text { Thick } \\
\end{array}$} & & & & \\
\hline & orean & $12 \%$ & Gneal $12 \%$ & & Wood Bark & & vol & Tang & Rid & & & & & & \\
\hline Redwood, Old Gro & & 0.40 & $53.8 \quad 28.0$ & 127 & 9,210 & 5,452 & & 4.4 & & 5.81 & 0.98 & 3.80 & 8.1 & 263 & $2,371.4$ \\
\hline Redwood, Young Growth & 0.30 & 0.35 & 425 & 127 & 9,210 & 5,452 & 7.0 & 4.9 & 2.2 & 5.78 & 0.98 & 3.79 & 6.4 & 264 & 1,8721 \\
\hline Spruce, Black & 0.38 & 0.40 & $429 \quad 28.0$ & 81 & $8,100 \quad 8,630$ & 4,599 & 11.3 & 6.8 & 4.1 & 5.70 & 0.97 & 3.69 & 7.9 & 271 & $2,371.4$ \\
\hline Spruos, Englemar & 033 & 035 & 13.7 & 112 & 3,100 & & 11.0 & 2.1. & 3.8 & 5.69 & 00.97 & 3.69 & 68 & 271 & $2059: 4$ \\
\hline Spruo,red & 836 & 038 & $40,7.266$ & 3 & $8,100 \% 8,630$ & & 129 & 74 & 40 & 567 & 0097 & 367 & 74 & 272 & 2246.6 \\
\hline Spruce Sita & 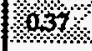 & 0.40 & 441,280 & t & $8,100,68,630$ & 45 & 115 & 2 & 43 & 5.68. & 0.97 & 3.8 & 7,6 & 273 & $2 ; 309.0$ \\
\hline Spruce, White & 0.37 & 0.40 & $41.8 \quad 28.0$ & 81 & $8,100 \quad 8,630$ & 4,599 & 115 & 7.2 & 4.3 & 5.68 & 0.97 & 3.67 & 7.6 & 273 & $2,309.0$ \\
\hline Yew, Pacific & 0.60 & 0.62 & 43.3 & 44 & 9,200 & 5.445 & 9.7 & 5.4 & 4.0 & 5.76 & 0.97 & 3.73 & 12.6 & 268 & $3,744.3$ \\
\hline Importhwods & \%. & & ঝ. & m & & & & \% & $\%$ & $2 \%$ & $\%$ & औ & $\begin{array}{l} \\
3 \\
3\end{array}$ & \begin{tabular}{cc} 
& \\
$\ddots$ & $\ddots$ \\
$\ddots$ & $\ddots$ \\
\hdashline & $\ddots$
\end{tabular} & $\begin{array}{l}\because \\
\because \vdots \\
\ddots \\
\vdots\end{array}$ \\
\hline Angelique & 0.60 & & 56.2 & 50 & & & & 8.8 & 5.2 & 5.61 & 0.96 & 3.60 & 12.1 & 278 & $3,744.3$ \\
\hline Balsa & 0.13 & 0.17 & 12.211 .9 & 50 & & & & 6.5 & 3.7 & 5.71 & 0.97 & 3.71 & 2.7 & 270 & 811.3 \\
\hline Banak & 0.42 & & 39.3 & 50 & & & & 8.8 & 4.6 & 5.61 & 0.97 & 3.62 & 8.5 & 277 & 2.621 .0 \\
\hline Grientheart & 0.83 & & 777 & $50:$ & & & & 60. & 82 & 560 & 8094 & 3 & 164 & 285 & $5,179,6$ \\
\hline Jefutong & 036 & & 337 & so & & & & & & 6,009 & 100 & 400 & $8.1 \%$ & 250 & $2,246.6$ \\
\hline Carch Europesi & 0,33 & & 30,9 & 50 & $\therefore$ & & & 53 & 31 & $5 \pi$ & 098 & 3.76 & $x_{0}$ & 266 & $2,059.4$ \\
\hline Lignumvitae & 0.98 & 1.09 & $91.7 \quad 76.2$ & 50 & & & & & & 6.00 & 1.00 & 4.00 & 22.0 & 250 & $6,115.7$ \\
\hline Lauan, Bagtikan & 0.48 & & 44.9 & 50 & & & & 8.0 & 3.8 & 5.65 & 0.97 & 3.66 & 9.9 & 273 & $2,995.4$ \\
\hline Lauan, Dark Red & 0.44 & & 41.2 & 50 & & & & 8.0 & 3.8 & 5.65 & 0.97 & 3.66 & 9.0 & 273 & $2,745.8$ \\
\hline Lanan, Light Red & $041:$ & 4. & $38.4,30.8$ & .50 . & & & & 8.0 & 38 & 5,65 & 0.97 & 3,66 & 34 & 273 & $2,558.6$ \\
\hline Launn, Mayapis & 0.41 & & 38.4 & so & & & & 80 & 38 & 5.65 & 0.97 & $3.65 \%$ & 8.4 & 273 & $2,558.6$ \\
\hline Lauan Red fanguile & 046 & & 43, & 50 & & & & 8,0 & 38 & 5,65 & 0097 & 366 & 95 & 273 & $2,870.6$ \\
\hline Lauan, White & 0.43 & & 40.2 & 50 & & & & 8.0 & 3.8 & 5.65 & 0.97 & 3.66 & 8.8 & 273 & $2,683.4$ \\
\hline Mahogany & 0.45 & & 42.1 & so & & & & 5.1 & 3.1 & 5.78 & 0.98 & 3.76 & 9.5 & 266 & $2,808.2$ \\
\hline Oak, English & 0.57 & & 53.4 & 50 & & & 16.7 & 10.7 & 5.1 & 5.53 & 0.96 & 3.55 & 11.4 & 282 & $3,557.1$ \\
\hline obecte: & 0.33 & & 309 & 50 & & & & 3 & 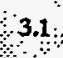 & sint & 0.98 & 3,36 & 70 & 266 & $2,059.4$ \\
\hline Paran Pine & 0.46 & & 43,10 & 50 & & & & 00 & & 5.65 & 097 & 3.66 & 94 & 273 & $2,870.6$ \\
\hline Pine; Caribbea & 0.68 & & $63.6 \%$ & 50 & & & & $\therefore$ & & $600 \%$ & 100 & $400 \%$ & $15 \%$ & 250 & $4,243.5$ \\
\hline Pine, Ocote & 0.55 & & 51.5 & 50 & & & & & & 6.00 & 1.00 & 4.00 & 12.4 & 250 & $3,432.3$ \\
\hline Ramin & 0.59 & & 55.2 & 50 & & & & 8.7 & 3.9 & 5.62 & 0.97 & 3.64 & 12.1 & 275 & $3,681.9$ \\
\hline Rosewood & 0.75 & & 70.2 & 50 & & & & & & 6.00 & 1.00 & 4.00 & 16.8 . & 250 & $4,680.4$ \\
\hline Spanishod Cedir & 034 & 0.36 & $318 \% 25.2$ & 50 & & & & 63 & & 5.72 & 097 & 3.10 & \%1 & 270 & $2,121.8$ \\
\hline Teak $\quad \vdots$ & 0,57 & 0.62 & 3344,43 & 50 & & & & 40 & 2 & 582 & 0.98 & 3,82 & $1+2$ & 262 & $3,557,1$ \\
\hline Walnut, European 1 & 0.47 & & $44.0 \mathrm{O}$ & 50 & 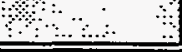 & 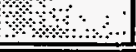 & & 6.4 & 43 & 5.72 & 0.97 & 3.69 & $9,7 \%$ & 271 & $2.933 .0^{\circ}$ \\
\hline
\end{tabular}




\section{Used Woods}

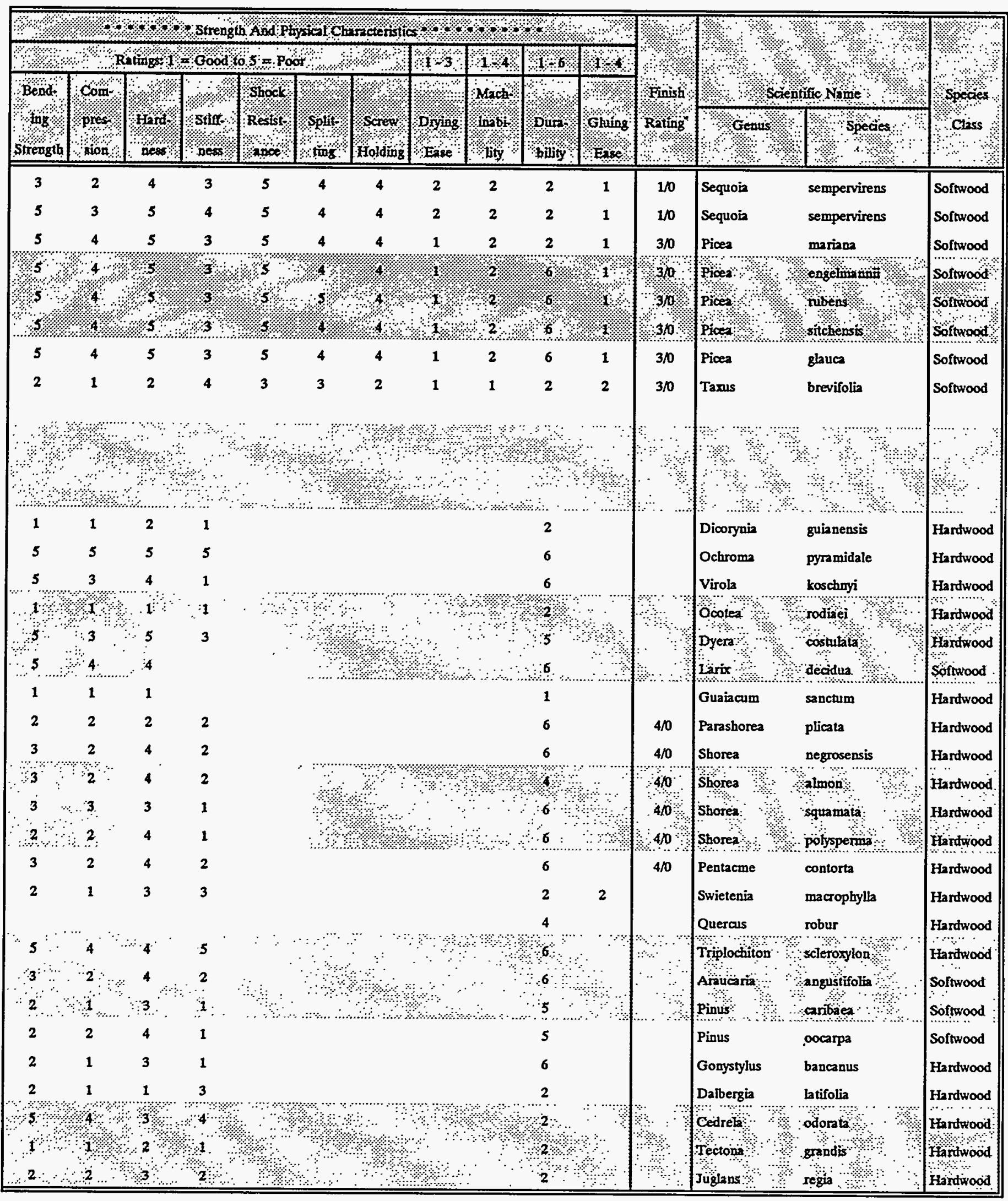




\section{REFERENCES}

Farmer, R.H. 1972. Handbook of Hardwoods, 2nd Edition. Princes Risborough Laboratory. Department of the Environment. Her Majesty's Stationery Office. London.

Forbes, Reginald D. 1961. Forestry Handbook. The Ronald Press Company. New York.

Green, David W. 1989. Moisture content and the shrinkage of lumber. Res. Pap. FPL-RP489. Forest Products Laboratory. USDA Forest Service. Madison, WI.

Haygreen, John G. and Jim L. Bowyer. 1982. Forest Products and Wood Science. The Iowa State University Press. Ames.

Howard, Alexander L. 1934. Timbers of the World. MacMillan and Co. London.

Howard, Elaine T. 1973. Untitled article. Wood Science. Vol 5, No. 3. pp. 194-197.

Liska, J.A. and J.T. Drow. 1973. Standard Terms for Describing Wood. Forest Products Laboratory. USDA Forest Service. Madison, WI.

Little, Elbert L., Jr. 1979. Checklist of United States Trees (Native and Naturalized). Agriculture Handbook No. 541. USDA Forest Service. Washington, D.C.

Panshin, A. J. and Carl de Zeeuw. 1970. Textbook of Wood Technology, Third Edition. McGraw-Hill Book Company. New York.

Rendle, B. J. 1969. World Timbers, Volume One. University of Toronto Press. Toronto, Ontario.

Rendle, B. J. 1969. World Timbers, Volume Two. University of Toronto Press. Toronto, Ontario.

Rendle, B. J. 1969. World Timbers, Volume Three. University of Toronto Press. Toronto, Ontario

Ross, Nancy. 1989. Complete Index of Common Names. Supplement to Tropical Timbers of the World. Forest Products Laboratory. USDA Forest Service. Madison, WI.

Toennisson, R. L. 1986. Common Wood Properties, Version 1.0. Forest Resources Development. Tennessee Valley Authority. Norris, TN.

Anonymous. 1965. Fine Hardwoods Selectorama. Fine Hardwoods Association. Chicago, IL.

Anonymous. 1974. Wood Handbook. Agriculture Handbook No. 72. Forest Products Laboratory. USDA Forest Service. Madison, WI.

Anonymous. 1991. Wood Properties Reports. The Luthier's Mercantile. Healdsburg, CA. 


\section{GLOSSARY}

BF - Board foot. A unit of lumber measurement equal to one foot square by one inch thick, or the equivalent of 144 cubic inches.

Btu - British thermal units. The quantity of heat required to raise the temperature of one pound of water by one degree Fahrenheit.

Commercial species - Tree species conventionally regarded as being able to develop into trees suitable for the manufacture of industrial timber products. Species which typically exhibit small size, poor form, or inferior quality are excluded.

Green Specific Gravity - Based on green volume and oven dry weight.

Hardwoods - Angiosperms; dicotyledonous trees, usually broadleaf and deciduous.

MBF - Thousand board feet.

MC - Moisture content. The percentage of moisture present in wood. $\%=$ original wt. oven dry wt. / oven dry wt. X 100.

ODW - Oven dry weight.

Rad - Radial.

Seasoned Specific Gravity - Based on volume at $12 \%$ moisture content and oven dry weight.

Tang - Tangential.

Softwoods - Gymnosperms; in the order Coniferales, usually evergreen (includes the genus Taxodium which is deciduous), having needles or scalelike leaves.

Vol - Volume. 


\section{NOTES}

